\title{
éditionsOCDE
}

\section{GUERRES ET CONFLITS AU SAHARA-SAHEL}

\section{NOTES OUEST-AFRICAINES}

Septembre 2017 No. 10 



\section{GUERRES ET CONFLITS AU SAHARA-SAHEL}

Cette note a été rédigée par

OLIVIER J. WALTHER

Groupe de recherche sur le Sahel, Université de Floride 


\section{NOTES OUEST-AFRICAINES}

La série Notes ouest-africaines analyse les dynamiques socio-économiques, politiques et sécuritaires que traverse l'Afrique dans une perspective régionale et multidisciplinaire. Elle cherche à stimuler la discussion, rassembler les informations et mieux anticiper les transformations en cours pour les politiques à venir. La série vise à partager des études avec une large audience d'experts, de praticiens du développement, de décisionnaires et de lecteurs avertis. Les Notes sont disponibles en anglais et/ou en français ; les résumés dans les deux langues. Initiées par le Club du Sahel et de l'Afrique de l'Ouest (CSAO) pour éclairer les enjeux ouest-africains, ces analyses sont préparées par son Secrétariat, ses membres et partenaires, les autres départements de l'OCDE, des organisations internationales et autres experts et chercheurs.

En savoir plus sur le Club du Sahel et de l'Afrique de l'Ouest : http://www.oecd.org/fr/csao.

Merci de citer cet ouvrage comme suit :

Walther, O. (2017), ( Guerres et conflits au Sahara-Sahel ), Notes ouest-africaines, N¹0, Éditions OCDE, Paris.

https://doi.org/10.1787/04bdd7aa-fr

Contact auteur : owalther@ufl.edu

ISSN 2415-1149

Les documents de travail de l'OCDE ne doivent pas être présentés comme exprimant les vues officielles de l'OCDE ou de ses pays membres. Les opinions exprimées et les arguments employés sont ceux des auteurs.

Ce document et toute carte qu'il peut comprendre ne préjugent en rien du statut de tout territoire, de la souveraineté s'exerçant sur ce dernier, du tracé des frontières et limites internationales, et du nom de tout territoire, ville ou région.

Les documents de travail exposent des résultats préliminaires ou des travaux de recherche en cours menés par l'auteur/les auteurs et sont publiés pour stimuler le débat sur un large éventail de questions sur lesquelles l'OCDE travaille. Les commentaires sur les documents de travail sont bienvenus et peuvent être adressés au Club du Sahel et de l'Afrique de l'Ouest, OCDE, 2 rue André-Pascal, 75775 Paris Cedex 16, France.

Autorisé pour publication par Laurent Bossard, Directeur, Secrétariat du Club du Sahel et de l'Afrique de l'Ouest (CSAO/OCDE).

(C) OCDE 2017

Vous êtes autorisés à copier, télécharger ou imprimer du contenu OCDE pour votre utilisation personnelle. Vous pouvez inclure des extraits des publications, des bases de données et produits multimédia de l'OCDE dans vos documents, présentations, blogs, sites Internet et matériel d'enseignement, sous réserve de faire mention de la source OCDE et du copyright. Les demandes pour usage commercial ou de traduction devront être adressées à rights@oecd.org. 


\title{
RÉSUMÉ
}

Le Sahel et le Sahara sont confrontés à une instabilité politique exceptionnelle mêlant rébellions, insurrections jihadistes, coups d'État, mouvements contestataires et trafics illicites. Cette combinaison de violences s'exprime aujourd'hui dans un environnement sécuritaire mondialisé, qui brouille les distinctions habituelles entre le local et le global, le militaire et le civil, la sphère domestique et la sphère internationale, la politique et l'identité. L'objectif de cette note est d'analyser ces dynamiques de violence. La première section décrit la distribution géographique et l'évolution temporelle des principaux foyers de violence d'Afrique du Nord et de l'Ouest. Elle discute ensuite les événements à l'origine de l'intensification de la violence politique au Sahel et au Sahara. La seconde section analyse ces dynamiques de violence, en s'attardant sur les échelles géographiques et les stratégies des belligérants. La note souligne en conclusion la nécessité de renforcer la coopération régionale, restaurer la légitimité des États et établir des solutions de gouvernance inclusives dans les zones de conflit.

Mots clés : sécurité, guerre, conflit, jihadisme, terrorisme, Sahara, Sahel

Classification JEL : D74, F5, H56, N47

\begin{abstract}
À PROPOS DE L'AUTEUR
Olivier J. Walther est professeur associé invité au Centre d'études africaines de l'Université de Floride et professeur associé en sciences politiques à l'Université du Sud-Danemark.

Titulaire d'un doctorat en géographie de l'Université de Lausanne, Dr Walther a orienté ses recherches sur le commerce, la coopération transfrontalière et le terrorisme en Afrique de l'Ouest en utilisant l'analyse des réseaux sociaux. Ses travaux ont été financés par la Commission européenne, le Programme alimentaire mondial, l'OCDE, l'Observatoire en réseau de l'aménagement du territoire européen, les gouvernements du Luxembourg et du Danemark, et la Fondation Carlsberg. Il est rédacteur pour l'Afrique du Journal of Borderlands Studies et membre du comité exécutif de l'African Borderlands Research Network (ABORNE).
\end{abstract}

\section{NOTE AUX LECTEURS}

Cette note actualise le chapitre « Enjeux sécuritaires, circulations et réseaux au SaharaSahel ») de l'Atlas du Sahara-Sahel publié par le Club du Sahel et de l'Afrique de l'Ouest de l'OCDE en 2014. Certaines parties de ce document s'appuient sur un blog rédigé par l'auteur et Antonin Tisseron en 2015 et sont reproduites avec l'autorisation de The Broker : ( Strange bedfellows: a network analysis of Mali's northern conflict »). 


\section{ACRONYMES}

ACLED Armed Conflict Location \& Event Data Project

ADC Alliance démocratique du 23 mai pour le changement

AIS Armée islamique du salut

ALS Armée de libération du Soudan

AND Alliance nationale démocratique

ANR Alliance nationale de la résistance

AQMI Al-Qaida au Maghreb islamique

ATNMC Alliance touareg nord Mali pour le changement

ClJ Cour internationale de justice

CJA Congrès pour la justice dans l'Azawad

CMA Coordination des mouvements de l'Azawad

CMFPR Coordination des mouvements et forces patriotiques de résistance

CNR Conseil national de la résistance

CNRT Conseil national de redressement du Tchad

CNT Concorde nationale tchadienne

CSNPD Conseil du sursaut national pour la paix et la démocratie

CPA Coalition du peuple pour l'Azawad

CRA Coordination de la résistance armée

EUTM Mali Mission de formation de l'Union européenne au Mali

FARF Forces armées pour la république fédérale

FARS Forces armées révolutionnnaires du Sahara

FDR Front démocratique du renouveau

FIAA Front islamique arabe de l'Azawad

FIS Front islamique du salut

FLAA Front de libération de l'Aïr et de l'Azawad

FLM Front de libération du Macina

FNTR Front national tchadien rénové

FONALT Forces des organisations nationales pour l'alternance et les libertés

FPA Front populaire de l'Azawad

FPR Front patriotique de résistance

FPRN Front populaire pour la renaissance nationale

FROLINAT Front de libération nationale du Tchad

FRRRT Force pour le ratissage, le regroupement et le redressement du Tchad

FUC Front uni pour le changement

FUCD Front uni pour le changement démocratique

GATIA Groupe autodéfense touareg Imghad et alliés

GCT Groupe combattant tunisien

GIA Groupe islamique armé

GICM Groupe islamique combattant marocain

GSL Groupe salafiste libre

GSPC Groupe salafiste pour la prédication et le combat
HCR Agence des Nations Unies pour les réfugiés

HCUA Haut Conseil pour l'unité de l'Azawad

JNIM Jama'a Nusrat ul-Islam wa al-Muslimin'

LIDD Ligue islamique pour le dawa et le jihad

LIFD Groupe islamique combattant en Libye

LRA Armée de résistance du Seigneur

MAA Mouvement arabe de l'Azawad

MDD Mouvement pour la démocratie et le développement

MDJT Mouvement pour la démocratie et la justice au Tchad

MIA Mouvement islamique de l'Azawad

MINURSO Mission des Nations Unies pour l'organisation d'un référendum au Sahara occidental

MINUSMA Mission multidimensionnelle intégrée des Nations Unies pour la stabilisation au Mali

MJE Mouvement pour la justice et l'égalité

MNJ Mouvement des Nigériens pour la justice

MNJTF Force multinationale conjointe

MNLA Mouvement national de libération de l'Azawad

MPA Mouvement populaire de l'Azawad

MPLA Mouvement populaire de libération de l'Azawad

MPS Mouvement patriotique du salut

MPSA Mouvement populaire pour le salut de l'Azawad

MSA Mouvement pour le salut de l'Azawad

MUJAO Mouvement pour l'unicité et le jihad en Afrique de l'Ouest

NTC Conseil national de transition

POLISARIO Front populaire de libération de la Saguia el Hamra et du Rio de Oro

PREGEC Prévention et gestion des crises alimentaires

RAFD Rassemblement des forces démocratiques

RDL Rassemblement pour la démocratie et la liberté

RFUTD Rassemblement des fils unis du Tchad pour le développement

RPJ Rassemblement populaire pour la justice

SCUD Socle pour le changement, l'unité et la démocratie

UFDD Union des forces pour la démocratie et le développement

UFR Union des forces de la résistance

UNESCO Organisation des Nations Unies pour l'éducation, la science et la culture 


\section{TABLE DES MATIÈRES}

RÉSUMÉ EXÉCUTIF

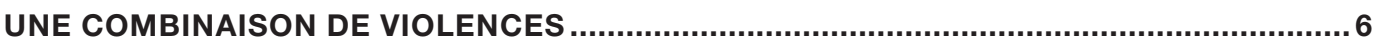

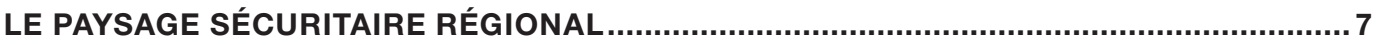

Principaux foyers de violence régionaux .......................................................................

L'instabilité politique croissante du Sahara-Sahel .........................................................10

SPÉCIFICITÉS DES GUERRES ET CONFLITS DU SAHARA-SAHEL ............................... 14

Des conflits locaux, globaux et transfrontaliers ........................................................... 14

Des alliances et rivalités très variables.........................................................................17

Des politiques identitaires exclusives ............................................................................ 19

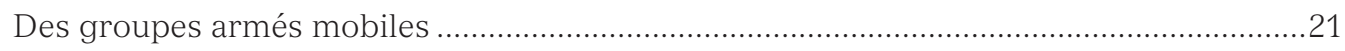

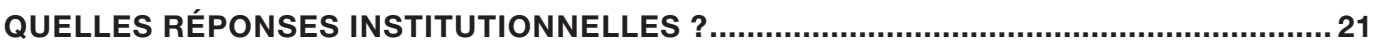

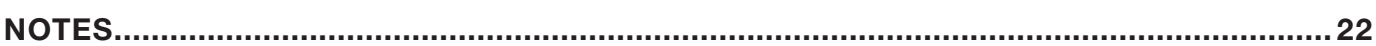

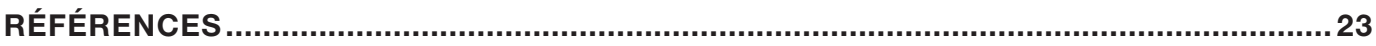

ANNEXE : GROUPES ARMÉS EN CONFLIT OUVERT AVEC LES ÉTATS SAHÉLOSAHARIENS, JANVIER 1990-DÉCEMBRE 2016.

\section{RÉSUMÉ EXÉCUTIF}

C ette note analyse les particularités de la situation sécuritaire du Sahel et du Sahara, confrontés à une instabilité politique exceptionnelle mêlant rébellions, insurrections jihadistes, coups d'État, mouvements contestataires et trafics illicites de drogue, d'armes et de migrants. Cette combinaison s'exprime aujourd'hui dans un environnement sécuritaire mondialisé, qui ne connaît plus de frontières strictes entre le local et le global, la sphère domestique et la sphère internationale, le militaire et le civil, la politique et l'identité.

Les conflits actuels ont en effet pour spécificité de mobiliser des ressources globales au profit de revendications locales. Les acteurs des conflits sahélo-sahariens exploitent la porosité des frontières nationales, en se relocalisant de manière opportuniste selon les moyens militaires déployés par les États de la région et leurs partenaires internationaux. Du point de vue social, les relations entretenues entre belligérants sont marquées par une grande flexibilité. Les groupes armés sont fragmentés en factions qui peuvent s'unir temporairement contre un ennemi commun et qui reflètent des divisions ethniques ou socio-économiques. L'absence de coalition de grande ampleur autour des forces gouvernementales, des rebelles ou des extrémistes religieux contribue à retarder les processus de paix, comme dans le conflit malien.

Nombre de conflits contemporains de la région apparaissent moins motivés par des considérations idéologiques que par des revendications identitaires visant à promouvoir une vision idéalisée du passé, comme celle du Califat. Cette vision politique s'appuie sur la peur, la haine et la destruction pour créer des espaces homogènes du point de vue ethnique ou religieux. L'une des conséquences de ces politiques identitaires est de tuer ou de faire fuir volontairement les civils, ce qui les distingue des guérillas plus anciennes, dont l'objectif était de se gagner les faveurs de la population locale. Les stratégies et tactiques militaires mises en œuvre pour atteindre cet objectif reposent sur une grande mobilité et sur un nombre restreint de combattants, dont les attaques visent moins la défense d'un territoire que le contrôle de sa population. 
La dégradation de la situation sécuritaire au cours des 15 dernières années montre que les solutions militaires devraient être définies et appliquées sur une base régionale. Le conflit malien a, de ce point de vue, servi de catalyseur à de nombreuses initiatives destinées à promouvoir la sécurité, la gouvernance et le développement dans la région. Ces « stratégies Sahel ») culminent aujourd'hui avec la mise en place d'une force militaire conjointe par les États du G5. L'Alliance pour le Sahel nouvellement créée devrait permettre de coordonner les initiatives, jusqu'ici dispersées, des principaux bailleurs dans la région.

En parallèle, il apparaît également que le règlement des conflits à long terme doit passer par une restauration de la légitimité des États, minée ( par le bas » par la multiplication de groupes armés. Les agendas contradictoires et les alliances imprévisibles de ces groupes conduisent à brouiller la distinction habituelle entre le gouvernement, l'armée nationale et la société qui constituait jusqu'alors l'un des piliers des États modernes de la région. Au cours des dernières décennies, les interventions militaires et les procédures judiciaires internationales se sont aussi souvent substituées, ( par le haut ), aux États lorsque ces derniers étaient incapables de défendre leur territoire, de maintenir l'ordre intérieur ou de protéger leur patrimoine culturel. Enfin, la légitimité des États sahélo-sahariens est soumise à une compétition accrue de la part des organisations non gouvernementales dont les actions, bien que nourries par des motivations humanitaires, se substituent « par le côté ») aux services publics, notamment lors de conflits.

Le règlement des crises nécessite aussi que des solutions de gouvernance inclusives soient trouvées à l'intérieur de chaque État. Le fait que les civils paient un prix si important dans les conflits actuels devrait inciter les pouvoirs publics à recourir à la force minimum nécessaire dans leurs opérations anti-insurrectionnelles. Une stratégie visant à protéger les populations civiles des violences et à gagner leur soutien est la plus à même de contrecarrer les stratégies des groupes extrémistes fondées sur la peur et l'exclusion. Cette stratégie peut passer par la mise en place de régions sécurisées dans lesquelles des formes alternatives de politique plus inclusives sont mises en place et par la reconnaissance des vertus de la diversité et du cosmopolitanisme, deux ingrédients indispensables pour lutter contre les politiques identitaires.

\section{UNE COMBINAISON DE VIOLENCES}

J enlèvement de plus d'une trentaine de touristes occidentaux dans le désert algérien en 2003 par le Groupe salafiste pour la prédication et le combat (GSPC) - devenu Al-Qaida au Maghreb islamique (AQMI) - marque le début d'une période d'instabilité politique exceptionnelle dans l’histoire récente du Sahel et du Sahara. De l'océan Atlantique au lac Tchad, il n'y a plus guère que le Sénégal qui échappe aux conflits armés ou au terrorisme. Cette situation s'explique par plusieurs dynamiques de violence qui conjuguent rébellions, insurrections jihadistes, attentats, prises d'otages, coups d'État, mouvements contestataires issus du Printemps arabe, et trafics illicites de drogue, d'armes et de migrants. Considérées séparément, aucune de ces dynamiques n'est réellement nouvelle dans la région. Les rébellions secouent le Sahara depuis le début de la période coloniale, alors que le jihadisme, encore plus ancien, a connu plusieurs vagues depuis la guerre sainte menée par Usman Dan Fodio dans le nord du Nigéria actuel en 1804. Les coups d'État et les manifestations politiques sont, quant à eux, aussi vieux que les États sahélo-sahariens eux-mêmes, alors que les trafics transsahariens, dont celui des esclaves, structurent le Sahara depuis l'époque médiévale.

Quelles sont alors les particularités de la situation sécuritaire contemporaine ? Le SaharaSahel est-il victime d'une simple intensification des guerres et conflits ${ }^{1}$ qui ont marqué la région au $\mathrm{XX}^{\mathrm{e}}$ siècle ou est-il devenu le terrain de luttes armées d'une nature différente? L'objectif de cette note est de contribuer à répondre à cette question cruciale non seulement pour la stabilité des États sahélo-sahariens mais aussi pour la sécurité des populations civiles, premières victimes de la violence contemporaine. L'analyse des dynamiques 
sociales et spatiales des conflits montre que la spécificité de la période actuelle est de combiner trois types de violence organisée dans un environnement mondialisé : les luttes armées qui visent la capture du pouvoir politique, les activités criminelles convoitant l'enrichissement individuel et les violations des droits de l'homme destinée à créer des espaces ethniques ou religieux homogènes.

L'histoire récente de l'Afrique subsaharienne, depuis la fin de la guerre froide, illustre dramatiquement cette combinaison de violences. Les conflits de la région des Grands Lacs, par exemple, ont opposé non seulement les États et leurs milices pour le contrôle du Burundi, de la République démocratique du Congo et du Rwanda, mais aussi des groupes criminels exploitant les richesses minières et énergétiques de l'Ituri et du Kivu, ainsi que d'innombrables responsables de crimes contre l'humanité, parmi lesquels les ( génocidaires ) rwandais. Ces conflits ont en outre mis en évidence la grande porosité qui existe entre sphère politique, économique et identitaire, les mêmes acteurs - y compris les représentants de l'État - pouvant rechercher le pouvoir, l'enrichissement personnel et l'épuration ethnique. Des dynamiques similaires sont observées au Libéria et en Sierra Leone, où les violences organisées par les États, les groupes criminels et les entrepreneurs identitaires sont particulièrement meurtrières au cours des années 90. Depuis une quinzaine d'années, le Sahel et le Sahara ne sont plus guère épargnés, particulièrement dans le nord malien, le Liptako-Gourma, la région du lac Tchad et le Darfour.

La première partie de cette note décrit la distribution géographique et l'évolution temporelle des principaux foyers de violence d'Afrique du Nord et de l'Ouest ${ }^{2}$. Elle discute ensuite les événements récents ayant conduit à une intensification de la violence politique dans la région du Sahel et du Sahara. La seconde partie analyse ces dynamiques de violence, en les replaçant dans un cadre qui dépasse les descriptions factuelles et l'apparente imprévisibilité des conflits de la région. La note se termine par plusieurs conclusions en matière d'action publique qui soulignent la nécessité de mettre en œuvre des réponses institutionnelles adaptées aux nouvelles échelles et combinaisons d'acteurs des conflits sahélo-sahariens.

\section{LE PAYSAGE SÉCURITAIRE RÉGIONAL}

es violences observées au cours des 20 dernières années en Afrique de l'Ouest et du Nord Lse concentrent dans plusieurs foyers de violence, liés aux insurrections soudanaises et nigérianes, ainsi qu'aux guerres civiles et manifestations populaires d'Algérie, d'Égypte et de Libye. L'évolution sécuritaire du Sahara-Sahel depuis les années 60 reflète quant à elle celle de l'Afrique dans son ensemble, marquée par une recrudescence des conflits impliquant des acteurs non étatiques depuis le début de la décennie 90 (Melander et al., 2016).

\section{Principaux foyers de violence régionaux}

Les quelque 243000 victimes civiles et militaires des violences observées entre 1997 et 2016 en Afrique de l'Ouest et du Nord dans la base de données de l'Armed Conflict Location \& Event Data Project (ACLED) (Raleigh et al., 2010 ; Raleigh et Dowd, 2015) sont très inégalement réparties géographiquement. La violence politique s'exerce dans un nombre restreint de microrégions plutôt qu'à l'échelle de pays entiers.

- Le plus grand foyer de conflit régional est soudanais. Près de la moitié des victimes de la région (109 160) meurent en effet des suites des violences exercées par l’État soudanais, ses milices alliées et les groupes armés du Darfour dans l'est du pays, ainsi que des violences liées au conflit du Soudan du Sud, qui précède et suit son accession à l'indépendance en 2011. À l'est comme au sud, la dimension transfrontalière est particulièrement évidente et se manifeste par un exode massif de réfugiés vers les pays voisins. 
- Le second foyer de conflit régional est d'origine nigériane. Il conjugue, à partir du début des années 2010, trois sources de violence de grande ampleur : l'insurrection jihadiste de Boko Haram ${ }^{3}$ dans la région transfrontalière du lac Tchad, les violences exercées par les groupes armés du Delta du fleuve Niger en lutte contre le gouvernement fédéral et les compagnies pétrolières, et les tensions ethniques entre pasteurs et agriculteurs dans le Centre (Middle Belt). Ces trois foyers représentent un quart des victimes civiles et militaires observées entre 1997 et 2016 dans la région (56 700 morts).

- Les autres épicentres de violence sont situés au nord du Sahara. En Libye, les tensions liées à la chute du Colonel Kadhafi en 2011 ainsi que les combats opposant milices islamistes et forces progouvernementales au cours de la guerre civile font 15500 morts sur la période considérée. En Algérie, l’essentiel des victimes est lié à la guerre civile qui oppose le gouvernement aux groupes islamistes jusqu'au début des années 2000 (plus de 13000 morts). En Égypte, la grande majorité des 10600 victimes observées résultent des violences faisant suite aux manifestations populaires du Printemps arabe débutant en 2011 et à la répression par le pouvoir militaire de la société civile et des associations religieuses (Société des Frères musulmans notamment).

Au cours des 20 dernières années, on observe des variations temporelles importantes, à l'exception de la région soudanaise, en conflit quasiment permanent depuis 1955 (Carte 1).

\section{Carte 1}

Les victimes de la violence en Afrique de l'Ouest et du Nord, 1997-2012
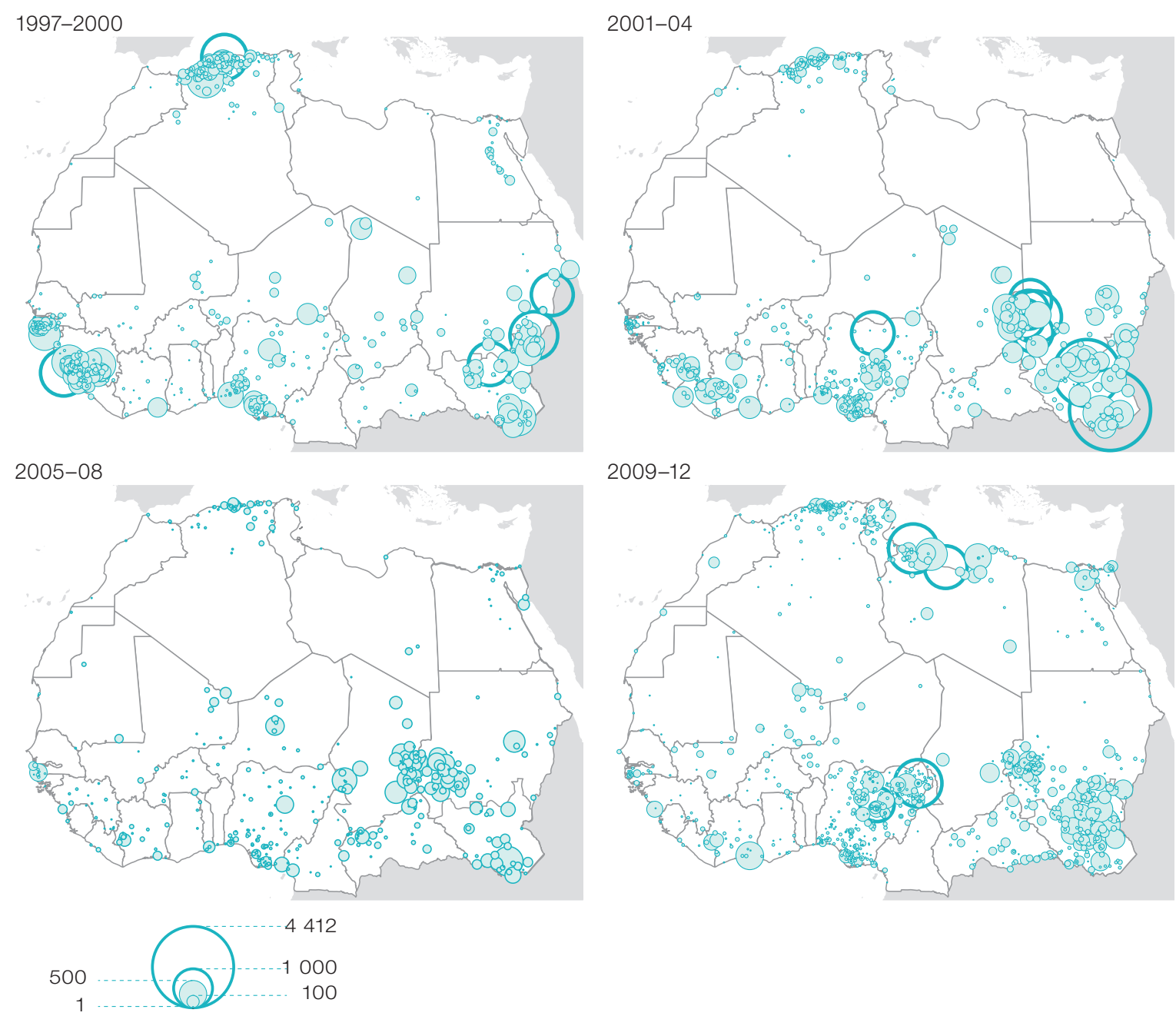

$2009-12$

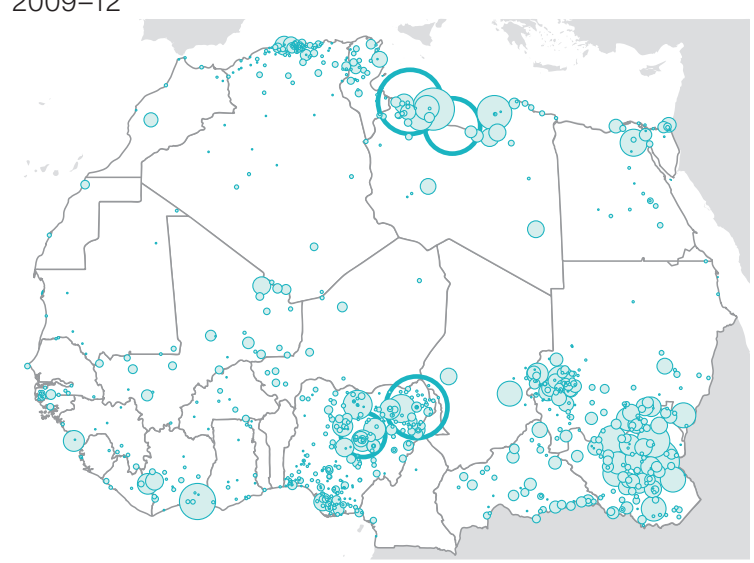

Source : ACLED, données 1997-2012 (www.acleddata.com/data/africa) 
Au sud du Sahara, le nombre élevé de victimes le long du golfe de Guinée dans les dernières années de la décennie 90 s'explique par les guerres civiles du Libéria (1989-97 et 1999-2003), de Sierra Leone (1991-2002) et de Guinée-Bissau (1998-99). À ces décès s'ajoutent ceux de la guerre civile algérienne (1991-2002), dont l'intensité diminue drastiquement avec la reddition de l'Armée islamique du salut (AIS) et la défaite du Groupe islamique armé (GIA) au début de la décennie 2000. Le nombre de victimes observées en Algérie passe ainsi de 3500 en 1997 à 130 en 2016 (Figure 1).

Figure 1

Nombre total de décès lors d'événements violents en Afrique de l'Ouest et du Nord, 1997-2016

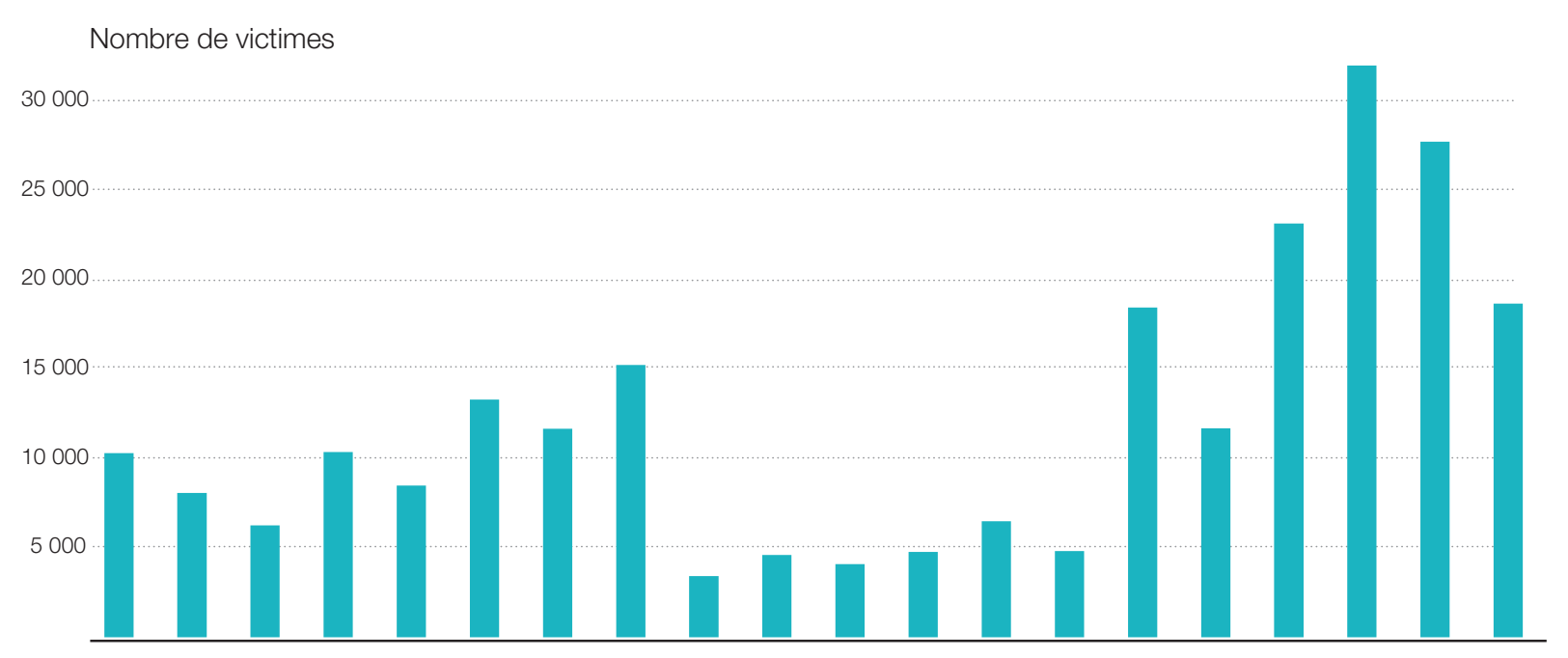

Source : ACLED, données 1997-2016 (www.acleddata.com/data/africa)

Carte 2

Les victimes de la violence en Afrique de l'Ouest et du Nord, 2013-16

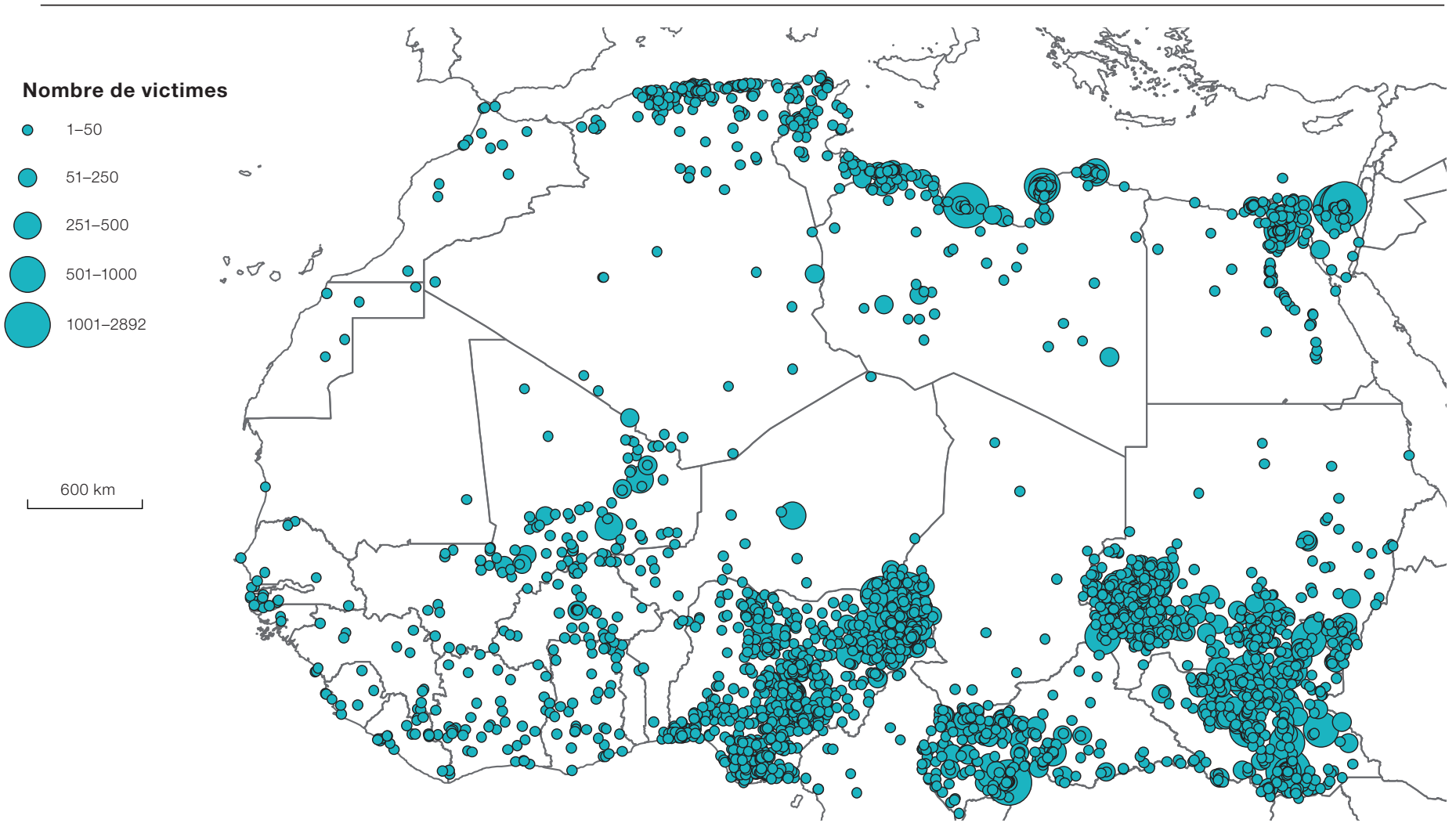

Source : ACLED, données 2013-16 (www.acleddata.com/data/africa) 
Le retour de la stabilité politique au Libéria et en Sierra Leone au début des années 2000 coïncide avec le début de la première guerre civile de Côte d'Ivoire (2002-07) et avec la montée en intensité du conflit du Darfour qui oppose l'Armée de libération du Soudan (ALS) et le Mouvement pour la justice et l'égalité (MJE) au gouvernement soudanais et aux milices arabes Janjaweed depuis 2003. Le nombre de victimes global connaît une diminution entre 2005 et 2010, avant d'augmenter significativement à partir de 2012 consécutivement aux conflits qui opposent rebelles et extrémistes religieux aux gouvernements de la région. La dégradation de la situation sécuritaire atteint son apogée en 2014 avec les effets conjugués du conflit malien qui débute en 2012, des guerres civiles de République centrafricaine et de Libye, des conflits du Darfour et du Soudan du Sud et des insurrections au Nigéria (Carte 2). La diminution du nombre de décès observée à partir de 2015 s'explique notamment par le repli de Boko Haram autour du lac Tchad.

\section{L'instabilité politique croissante du Sahara-Sahel}

Au cours des 50 dernières années, le Sahara-Sahel connaît plusieurs cycles de violence durant lesquelles la souveraineté des États est contestée par des rivalités interétatiques et des juntes militaires mais également, et de manière croissante, par des acteurs non étatiques aux revendications autonomistes, indépendantistes ou religieuses.

Les 15 années qui suivent les indépendances des États sahélo-sahariens (1960-75) sont caractérisées par une certaine stabilité qui contraste, rétrospectivement, avec la période contemporaine. Bien que la région connaisse plusieurs coups d'État qui instaurent des régimes autoritaires au Mali, au Niger, au Tchad et en Libye, les relations entre États nouvellement indépendants sont relativement pacifiées et peu de conflits ouverts sont enregistrés. Le nord du Mali connaît néanmoins sa première rébellion touareg (1962-64), brutalement réprimée par le gouvernement de Modibo Keïta, tandis que le Front de libération nationale du Tchad (FROLINAT) naît en 1966 au Soudan.

Les années 1975-90 sont quant à elles troublées par deux grands conflits régionaux. À l'extrémité occidentale du continent, le conflit du Sahara occidental oppose le Maroc et la Mauritanie au Front populaire de Libération de la Saguia el Hamra et du Rio de Oro (Polisario), allié de l'Algérie, à partir de 1976. La construction de plusieurs murs défensifs par le Maroc entre 1980 et 1987 (Carte 3) et le cessez-le-feu signé sous l'égide de la Mission des Nations Unies pour l'organisation d'un référendum au Sahara occidental (MINURSO) en 1991 consacrent le contrôle de facto du Maroc sur la majeure partie du Sahara occidental. À l'est, la Libye et le Tchad s'affrontent au sujet de la bande d'Aouzou, envahie par les forces libyennes en 1973 (Carte 4). Après un cessez-le-feu signé en 1987, la souveraineté tchadienne sur la bande d'Aouzou est reconnue par la Cour internationale de justice (CIJ) mettant un terme au conflit en 1994. La décennie 80 est également synonyme de plusieurs disputes frontalières réglées par la CIJ, entre le Burkina Faso et le Mali pour le contrôle de la bande d'Agacher (1985-86) et entre le Nigéria et le Cameroun dans la région du lac Tchad et la Péninsule de Bakassi (1987-2002).

Les années 90 marquent le début d’une nouvelle période d'instabilité caractérisée par une augmentation des conflits mettant aux prises États, rebelles et extrémistes religieux. Entre 1990 et 2016, par exemple, pas moins de 20 groupes armés majeurs étaient en conflit ouvert avec l'État malien (Annexe), à commencer par le Mouvement populaire de libération de l'Azawad (MPLA), dont la rébellion lancée dès les premières années de la transition démocratique s'achève par la signature d'un Pacte national destiné à favoriser l'intégration des Touareg dans la société malienne en 1996. À la même période, une rébellion met aux prises le gouvernement nigérien à plusieurs groupes touareg dont le Front de libération de l'Aïr et de l'Azawad (FLAA) et la Coordination de la résistance armée (CRA). Après de nombreuses exactions commises par les parties gouvernementales et rebelles, les hostilités prennent fin avec les accords de Ouagadougou en 1995. 


\section{Carte 3}

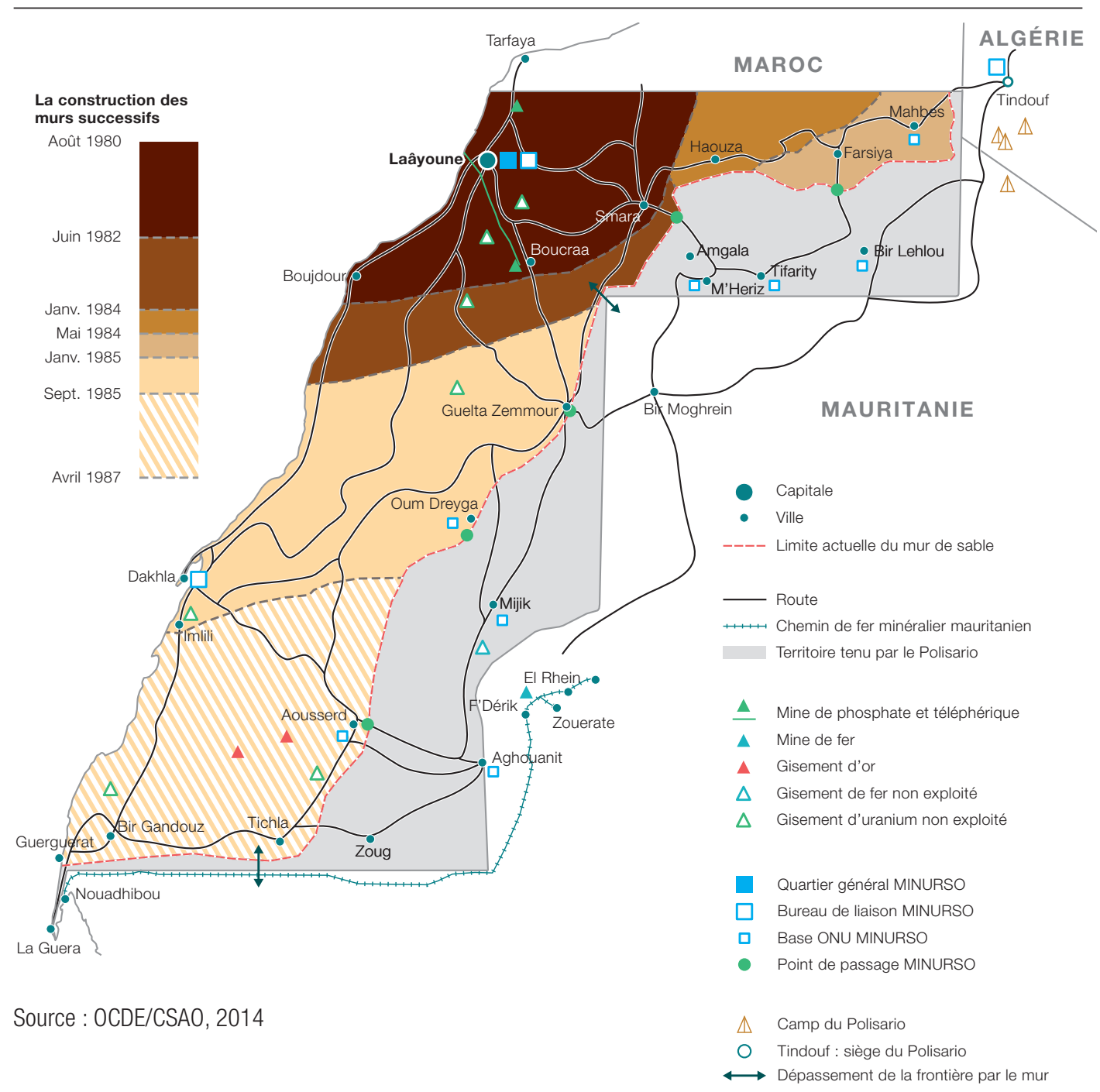

Une seconde vague de rébellions secouent la région à partir du milieu des années 2000. Au Mali, plusieurs leaders touareg des rébellions précédentes rallument les revendications indépendantistes en attaquant Kidal et Menaka en 2006. Les Accords d'Alger signés la même année mettent provisoirement un terme à la violence, avant qu'une nouvelle rébellion, conduite notamment par l'Alliance démocratique du 23 mai pour le changement (ADC) au Mali et le Mouvement des Nigériens pour la justice (MNJ) au Niger ne déstabilisent à nouveau la région entre 2007 et 2009.

Ces conflits, fréquemment interrompus par une série d'accords précaires, affectent différemment l'équilibre politique interne des États sahélo-sahariens. Au Niger, l'intégration des anciens rebelles à l'armée nationale et aux collectivités locales issues de la décentralisation conduisent à un apaisement des tensions sahariennes (Grégoire, 2013) . Au Mali, au contraire, la politique du Président Amadou Toumani Touré (2002-12) visant à gouverner le Nord par l'intermédiaire de tribus touareg alliées conduit à un retrait des institutions étatiques, à la prolifération des trafics transsahariens et à l'installation de groupes extrémistes à partir du milieu des années 2000. À la même période, la guerre civile libyenne consécutive à la chute du Colonel Kadhafi en 2011 crée un nouveau foyer d'instabilité au nord du Sahara. Elle conduit de nombreux mercenaires touareg de l'armée libyenne à rentrer au Mali pour prendre part à une nouvelle rébellion et à une prolifération du trafic d’armes légères en provenance des arsenaux libyens (Marsh, 2017). 
Carte 4

Les guerres du Tchad, 1973-2002

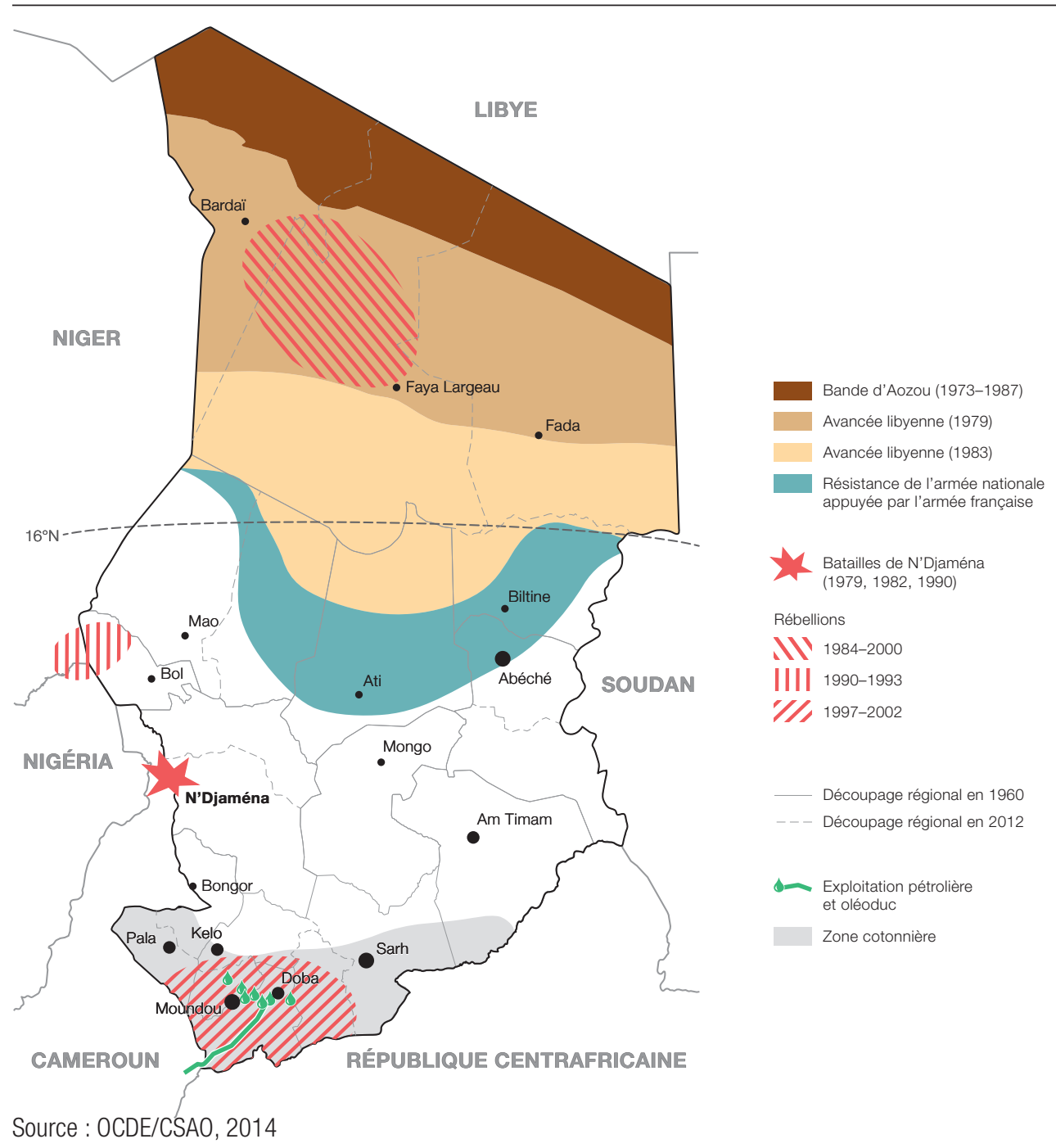

En janvier 2012, le développement de l'extrémisme religieux prend une dimension encore inédite, suite à l'alliance circonstancielle entre le groupe extrémiste Ansar Dine et les rebelles touareg du Mouvement national de libération de l'Azawad (MNLA). En quelques semaines, le nord du Mali et ses villes-clés sont conquis et le gouvernement du Président Touré remplacé par une junte militaire. La dégradation de la situation sécuritaire conduit le Conseil de sécurité des Nations Unies à autoriser le déploiement d’une force internationale au Mali en décembre 2012. En janvier 2013, une nouvelle offensive des extrémistes religieux en direction de Mopti convainc le Président malien par intérim, Dioncounda Traoré, de demander l'intervention de l'armée française qui lance l'Opération Serval. À l'issue d'une opération d'une ampleur encore inégalée depuis la guerre d’Algérie, l'armée française, appuyée par des troupes maliennes et tchadiennes, reconquiert rapidement le nord du Mali et détruit les bases d'AQMI, du Mouvement pour l'unicité et le jihad en Afrique de l'Ouest (MUJAO) et d'Ansar Dine à Tombouctou, Gao, Kidal et dans le massif des Ifoghas. En rétablissant l'intégrité territoriale du Mali, elle ouvre la voie au déploiement de la Mission multidimensionnelle intégrée des Nations Unies pour la stabilisation au Mali (MINUSMA) et à la Mission de formation de l’Union européenne au Mali (EUTM Mali).

Les forces militaires françaises se réorganisent sous l'égide de l'Opération Barkhane visant à lutter contre les groupes terroristes à partir d'août 2014 (Griffin, 2016) tandis qu'une solution politique du conflit malien tarde à se concrétiser. Près de cinq ans après la reconquête du nord du Mali, l'insécurité nourrie par les rivalités entre anciens rebelles, 
extrémistes religieux et éléments de la force internationale demeure préoccupante. Malgré le succès initial de l'opération militaire Serval, les forces maliennes, africaines et internationales présentes au Mali sont régulièrement la cible d'attaques d'Al-Mourabitoun, Ansar Dine, AQMI et du Front de libération du Macina (FLM), réunis sous l'appellation Jama'a Nusrat ul-Islam wa al-Muslimin' (JNIM) depuis mars 2017. Plusieurs attentats terroristes frappent le sud du pays tandis que l'autorité de l'État malien reste fictive dans nombre de juridictions du nord où sont encore présents les ex-rebelles touareg. La cartographie des attaques entreprises par les principaux groupes extrémistes dans la région depuis le début de la guerre du Mali en 2012 illustre l'activité transnationale d'AQMI et de ses groupes affiliés (Al-Mourabitoun, les Signataires par le sang) et de Boko Haram (Carte 5).

Carte 5

Victimes des principaux groupes terroristes, 2012-16

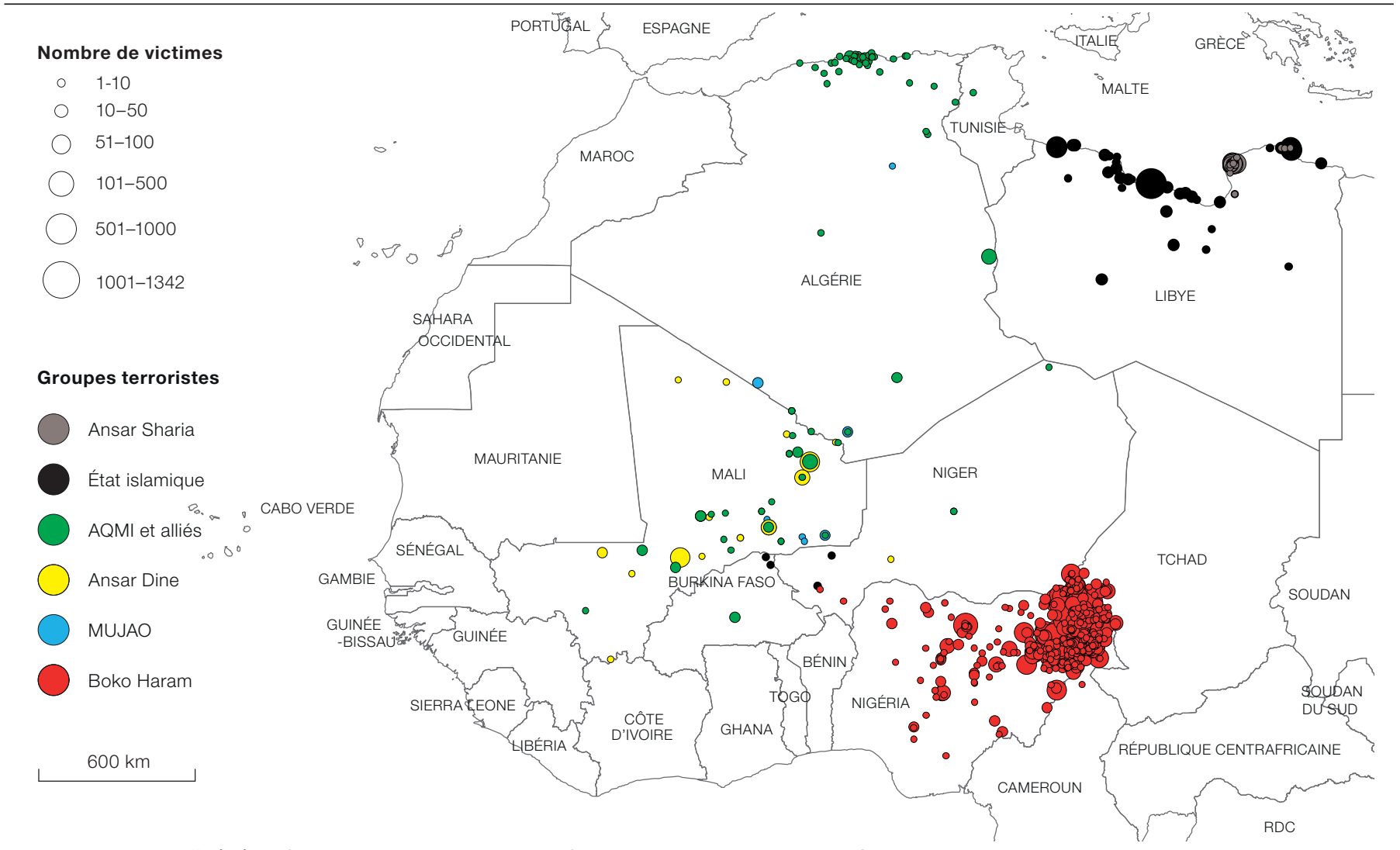

Note : les groupes affiliés à Al-Qaida au Maghreb islamique (AQMI) sont Al-Mourabitoun et les Signataires par le sang.

Seuls les événements violents dont l'attaquant est identifié sont pris en considération.

Source : ACLED, données 2012-16 (www.acleddata.com/data/africa).

Loin de se limiter aux confins sahariens, l'instabilité croissante qui caractérise le tournant des années 2010 se diffuse en effet également aux pays riverains du lac Tchad. La région est confrontée à une insurrection jihadiste particulièrement meurtrière, née des revendications de la secte Boko Haram, originellement centrée sur la ville de Maiduguri, dans l'État nigérian de Bornou, dont l'objectif est l'instauration (ou la restauration) d'un État islamique au Nigéria. Depuis la mort de son dirigeant Mohammed Yusuf en 2009, Boko Haram connaît un développement spectaculaire, tant du point de vue de son expansion territoriale que du nombre de victimes, civiles et militaires, liées à ses activités. Les efforts militaires déployés par les pays riverains du lac Tchad et leurs partenaires internationaux à partir de 2013 conduisent à une diminution du nombre d'attaques et de victimes significative depuis 2015 (ICR, 2016) (Figure 2). 
Figure 2

Victimes de violence par pays, 1997-2016

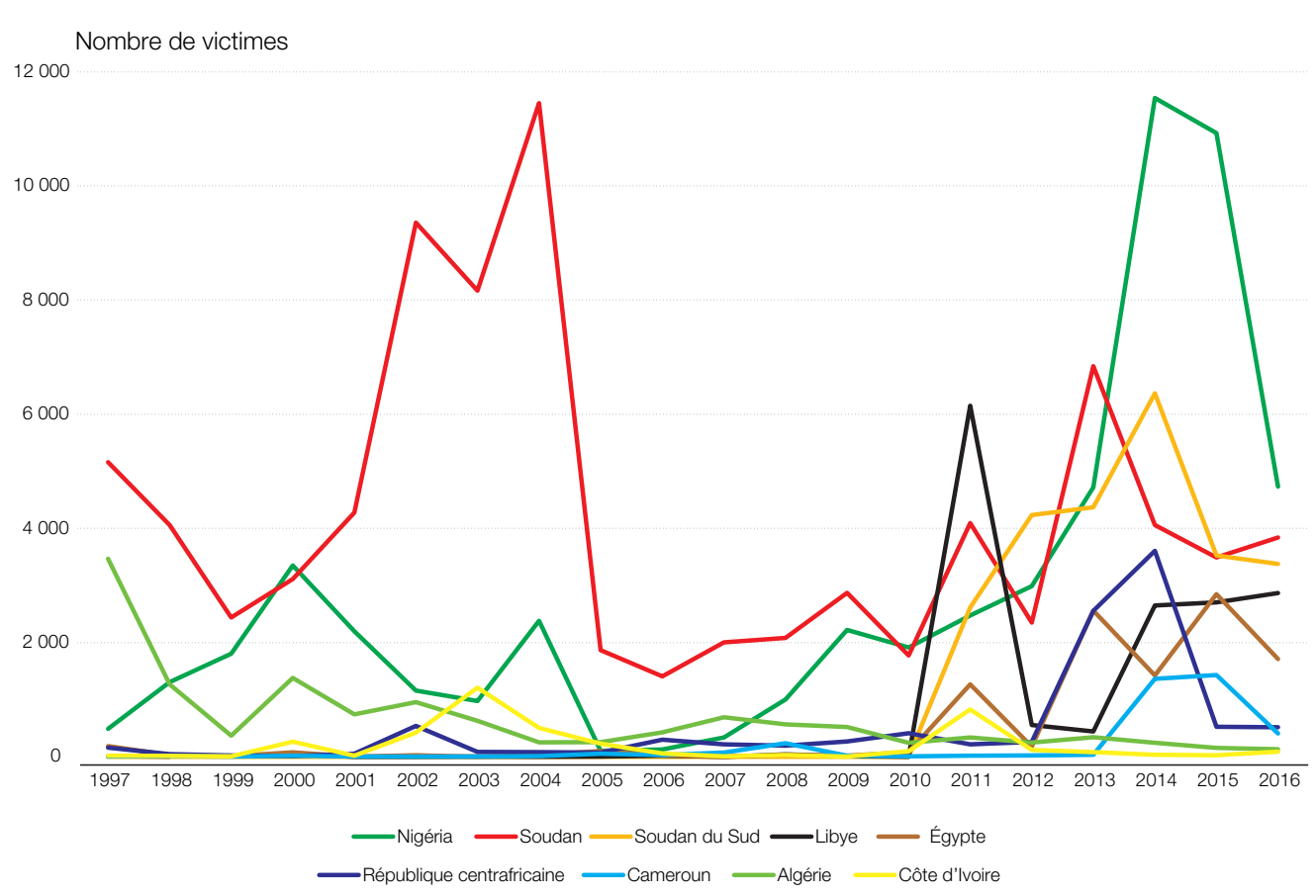

Note : seuls les neuf pays comptant le plus grand nombre de victimes sont représentés. Les événements violents pris en compte regroupent les catégories "batailles ", "violences contre civils ", " émeutes et manifestations ", et « violence à distance ».

Source : ACLED, données 1997-2016 (www.acleddata.com/data/africa).

Les conflits récurrents observés entre États et organisations non étatiques s'ajoutent aux désordres internes aux États sahélo-sahariens, qui sont loin d'avoir disparu depuis la fin de la guerre froide. Huit coups d'État réussissent depuis le début des transitions démocratiques dans les années 90, en Mauritanie (2005, 2008), au Niger (1996, 1999, 2010), au Tchad (1990) et au Mali (1991, 2012). Bien que faisant peu de victimes, ceux-ci déstabilisent les institutions démocratiques et encouragent les élites présidentielles à investir dans une garde rapprochée plutôt que dans les armées en charge de la sécurité nationale. Au Mali et au Burkina Faso, ces corps d'élite chargés de la sécurité présidentielle (commandos parachutistes et régiment de sécurité présidentielle) sont d'ailleurs réintégrés dans l'armée ou dissous. Aux coups d'État s'ajoutent les mouvements de contestation populaires qui débutent à la fin 2010 et se traduisent par une recrudescence de la violence politique et de l'extrémisme religieux en Tunisie et en Libye ainsi qu'à des manifestations de grande ampleur en Mauritanie.

\section{SPÉCIFICITÉS DES GUERRES ET CONFLITS DU SAHARA-SAHEL}

oin d'être totalement imprévisibles et chaotiques, les violences qui frappent le SaharaSahel répondent à plusieurs principes organisateurs qui permettent d'éclairer l'échelle géographique des conflits, les relations entre belligérants, leurs objectifs et leurs stratégies militaires.

\section{Des conflits locaux, globaux et transfrontaliers}

Du point de vue géographique, la première caractéristique des conflits sahélo-sahariens est qu'ils mobilisent des idées et des ressources globales au profit de revendications locales ou nationales. 
Boko Haram, par exemple, exploite un ensemble de récits historiques liés à l'empire du Kanem-Bornou, qui régna sur le bassin du lac Tchad et son prolongement saharien pendant près de 1000 ans, afin d'inscrire ses revendications politiques dans une continuité historique interrompue par la conquête coloniale (Barkindo, 2016). Cet ancrage dans l'histoire locale permet au groupe de justifier son projet de rénovation politique et de rejet des structures institutionnelles liées à la colonisation, dont la République fédérale du Nigéria. Simultanément, Boko Haram exploite la vision panislamiste d’un monde musulman unifié, dont les limites transcendent les frontières nationales pour s'appliquer à l'ensemble des croyants. Dans cette perspective, le groupe prête allégeance à l'État islamique en 2015, sans toutefois en récolter des bénéfices substantiels du point de vue opérationnel (Gartenstein-Ross et Zenn, 2016). La cartographie révèle une certaine correspondance entre l'extension de l'empire du Kanem-Bornou médiéval et les attaques conduites par Boko Haram depuis 2009, date à laquelle le groupe se tourne vers la violence politique (Carte 6). Selon les données d'ACLED, les 2074 attaques recensées, responsables de plus de 28000 victimes, sont particulièrement importantes dans l'État de Bornou, à Maiduguri (307), Damboa (57), Damaturu (49), Bama (49), ainsi que dans la métropole de Kano (49) et les villes-jumelles de Gambaru et Fotokol (43) à la frontière camerounaise.

\section{$\underline{\text { Carte } 6}$}

Empire du Kanem-Bornou et attaques de Boko Haram, 2009-16

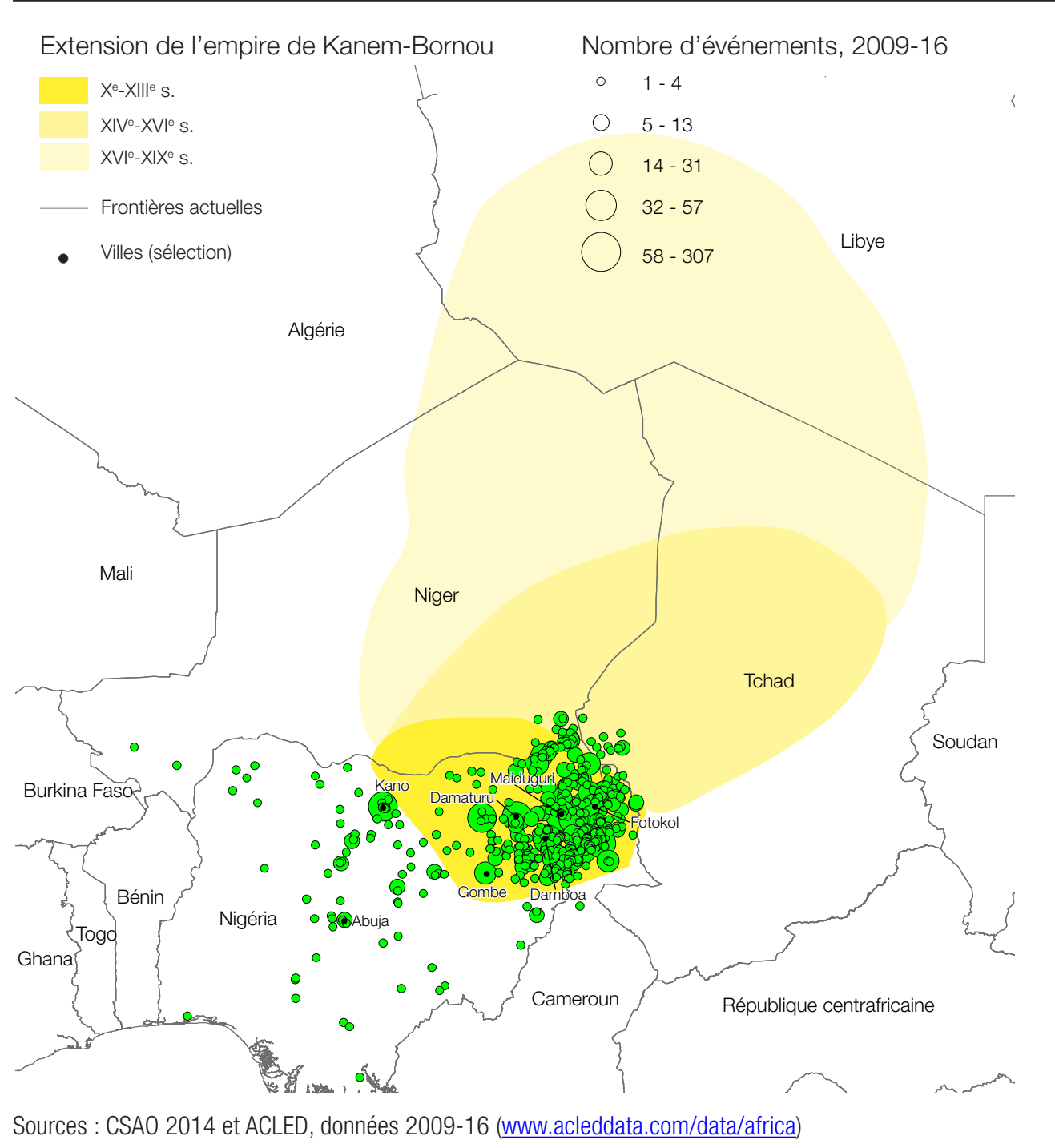


D'une manière similaire, les guerres et conflits de la région sont alimentés par des ressources globales qui sont ensuite investies dans des luttes aux aspirations locales ou nationales. Les initiateurs des conflits du Sahara-Sahel développent des stratégies prédatrices qui se nourrissent à la fois des ressources locales quand elles existent (pêche autour du lac Tchad), des flux de personnes et de marchandises transsahariens, souvent illicites et des ressources plus globales mises à disposition par les organisations humanitaires. Si dans d'autres régions d'Afrique subsaharienne, les camps de réfugiés et les ressources alimentaires mis à disposition par les organisations humanitaires sont utilisés par les rebelles pour prolonger leur lutte, il semble qu'au Sahara-Sahel ce sont essentiellement les otages européens qui permettent aux groupes extrémistes religieux de s'équiper en armes et en véhicules et à acheter les faveurs des chefs touareg et arabes locaux. Tout particulièrement au Mali, les rançons payées officieusement par les gouvernements en échange des otages européens représentent des sommes estimées à plusieurs dizaines de millions de dollars (Daniel, 2012).

La seconde manifestation géographique des conflits sahélo-sahariens est leur capacité à échapper aux cadres stricts des frontières d'État, brouillant la distinction habituelle entre les affaires domestiques et étrangères (Walther et Miles, 2018). De l'Atlantique à l'Ennedi, tous les États sahélo-sahariens ont renforcé la surveillance de leurs espaces frontaliers afin de lutter contre la prolifération des groupes rebelles ou terroristes. Cette situation s'observe dans les régions frontalières éloignées des capitales, comme le Hodh mauritanien, la forêt de Wagadou au Mali, le Gourma burkinabè, le Djado nigérien et le bassin du lac Tchad. Ces efforts ont reçu le soutien de l'Opération Barkhane, dont l'une des composantes est de déstabiliser les réseaux terroristes transfrontaliers dans certaines des régions les plus inaccessibles du Sahara. Dans certains cas, les forces gouvernementales ont usé de leur droit de poursuite pour intervenir dans un État voisin : la Mauritanie a entrepris des opérations militaires visant à détruire des bases d'AQMI situées en territoire malien (Harmon, 2014) avec le concours de l'armée française. Dans la région du lac Tchad, la menace régionale de Boko Haram a également conduit le Cameroun, le Niger, le Nigéria et le Tchad à revitaliser la Force multinationale conjointe (connue sous son acronyme anglais MNJTF), une structure de coopération conçue à l'origine pour lutter contre le banditisme et aujourd'hui principalement axée sur la lutte anti-terroriste.

Pour les rebelles et les extrémistes religieux, les frontières poreuses d'Afrique sahélosaharienne représentent une opportunité de circuler relativement facilement entre les différents pays de la région pour y conduire des attaques, s'approvisionner en armes ou recruter de nouveaux combattants. Comme au Libéria et en Sierra Leone, ou dans la vaste région forestière d'Afrique centrale qui abrite l'Armée de résistance du Seigneur (LRA en anglais), les zones frontalières du Sahel et du Sahara servent de base arrière pour des groupes désireux de se prémunir contre les actions offensives des États. L'histoire récente de la région montre à ce propos que la régionalisation des conflits suit le principe des vases communicants : soumis à la pression croissante, les groupes armés se relocalisent dans les pays où les capacités militaires ou la volonté politique de lutter contre eux sont les moins fortes.

À partir du milieu des années 2000, le nord malien ainsi devient le refuge du GSPC, dont les origines remontent au conflit qui oppose le gouvernement algérien à divers groupes islamistes entre 1991 et 2002. Soumis à une pression croissante de l'armée algérienne, le GSPC se déploie dans les provinces sahariennes d’Algérie et les régions frontalières voisines. En 2003, le groupe réussit une spectaculaire prise d'otages européens dans le désert algérien, s'installe dans le nord malien puis traverse le désert nigérien jusqu'au Tchad où son leader Amari Saifi (aussi connu sous le nom d'Abderrazak le Para) est capturé par les rebelles du Mouvement pour la démocratie et la justice au Tchad (MDJT). Entre 2004 et 2011, la mobilité d'AQMI et des autres groupes extrémistes circulant entre Mauritanie, Algérie, Mali, Niger et Tchad est à son apogée (Walther et Miles, 2018). Elle permet à leurs dirigeants de tisser des liens avec les tribus locales, de développer une industrie de l'enlèvement et de recruter un nombre croissant de combattants locaux d'origine touareg, arabe, peul et songhaï. 
Boko Haram connaît, au cours de la dernière décennie, une évolution comparable, passant d'un groupe militant principalement motivé par des objectifs nationaux à un réseau international présent dans un nombre croissant de pays (Dowd, 2018). De 2009 à 2012, alors que l'insurrection croît en intensité et s'étend géographiquement à partir de Maiduguri jusqu'à affecter 19 États du pays, l'immense majorité des attaques du groupe a lieu au Nigéria (99 \%). Un changement de stratégie s'opère à partir de 2014, année durant laquelle les attaques commises au Cameroun représentent 16 \% du total. En 2015, qui marque le pic de l'insurrection, plus de $28 \%$ des 597 attaques du groupe sont commises en dehors du Nigéria. Depuis lors, frappé dans ses capacités opérationnelles et contraint de relâcher son contrôle territorial, Boko Haram semble avoir adopté une stratégie de relocalisation opportuniste dans les pays voisins du Cameroun, du Niger et du Tchad, rendant l'effort conjoint de la MNJTF toujours plus crucial.

\section{Des alliances et rivalités très variables}

Un autre aspect déconcertant des conflits du Sahara-Sahel est d'opposer un petit nombre d'acteurs de natures très différentes. Outre les forces gouvernementales et les milices qui leur sont alliées, ces conflits mettent aux prises des groupes d'autodéfense basés sur l'appartenance ethnique ou religieuse, des groupes rebelles en lutte pour l'indépendance ou pour une plus large autonomie, des organisations terroristes, des chefs de guerre et des criminels. Les relations entre ces acteurs sont caractérisées par des alliances et des rivalités très variables (Bencherif et Campana, 2016). Au début du conflit malien, par exemple, le groupe islamiste Ansar Dine et le mouvement indépendantiste touareg du MNLA forgent une alliance précaire afin de conquérir les principales villes du Mali. Parvenus à leur fin, ces deux protagonistes aux intérêts fondamentalement divergents se sont ensuite violemment combattus.

Les mouvements de rébellions sont souvent fragmentés en factions concurrentes qui reflètent des divisions tribales, ethniques, sociales et économiques au sein des sociétés sahélo-sahariennes. Les tribus touareg d'origine noble, comme les Ifoghas du nord-est du Mali, par exemple, entretiennent des relations conflictuelles à la fois avec d'autres tribus d'origine vassale (Imghad) et avec des tribus nobles d'autres régions. Ces rivalités se retrouvent dans les lignes de fracture qui divisent les mouvements d'opposition et les empêchent de s'unir au niveau supranational. Ainsi, en dépit d’un héritage culturel et de revendications autonomistes similaires, les rebelles touareg du Mali et du Niger n’ont jamais fusionné. Depuis les grandes sécheresses des années 70, de fortes inégalités économiques se développent également entre une minorité enrichie par la migration, le mercenariat ou les trafics transsahariens et le reste de la population dépendant du pastoralisme et du tourisme, deux secteurs touchés par l'instabilité climatique et politique.

Il n'est pas certain que ces divisions internes puissent un jour être dépassées par un projet idéologique, comme la restauration d'un ordre théologique, tant il est vrai que nombre de groupes armés mêlent revendications panislamistes et revendications locales. Le groupe Ansar Dine, dont le nom signifie Défenseurs de la religion, s'appuie par exemple sur un agenda religieux visant l'ensemble des Musulmans tout en étant principalement composé de représentants des tribus touareg d'origine noble. De ce point de vue, les rébellions et insurrections actuelles du Sahara-Sahel diffèrent très nettement des mouvements d'opposition d'inspiration maoïste qui se sont développés durant la guerre froide et dont la force reposait dans une structure militaire hiérarchique parfaitement intégrée à un appareil politique (Knoke, 2013).

La cartographie des relations entretenues entre les 15 belligérants principaux du conflit malien en 2015 confirme que les relations conflictuelles dominent très nettement les coalitions dans la région. La position structurelle du MNLA est, de ce point de vue, particulièrement précaire, du fait que le mouvement entretient des relations conflictuelles aussi bien avec l'État malien qu'avec les extrémistes religieux (depuis 2013) et le Groupe autodéfense touareg Imghad et alliés (GATIA) d’El Hadj Gamou (Figure 3). Les groupes islamistes, sans cesse recomposés, comptent aussi de nombreux ennemis. 
La cartographie des relations de coopération entre les acteurs du confit malien met quant à elle en évidence l'extrême fragmentation du paysage sécuritaire et l'isolation du gouvernement malien, qui ne peut compter que sur le soutien d'une milice et des Nations Unies. Les organisations islamistes affiliées à Al-Qaida forment, malgré leurs rivalités, un cluster d'alliances plus dense, alors que les rebelles sont divisés en deux camps selon les relations qu'ils entretiennent avec le gouvernement. Forces gouvernementales, rebelles et islamistes forment trois composants d'un environnement sécuritaire dans lequel aucun pôle n'est réellement capable de rallier l'un ou l'autre camp et d'assurer un règlement durable du conflit.

Figure 3

Relations de conflit et de coopération entre acteurs du conflit malien en 2015

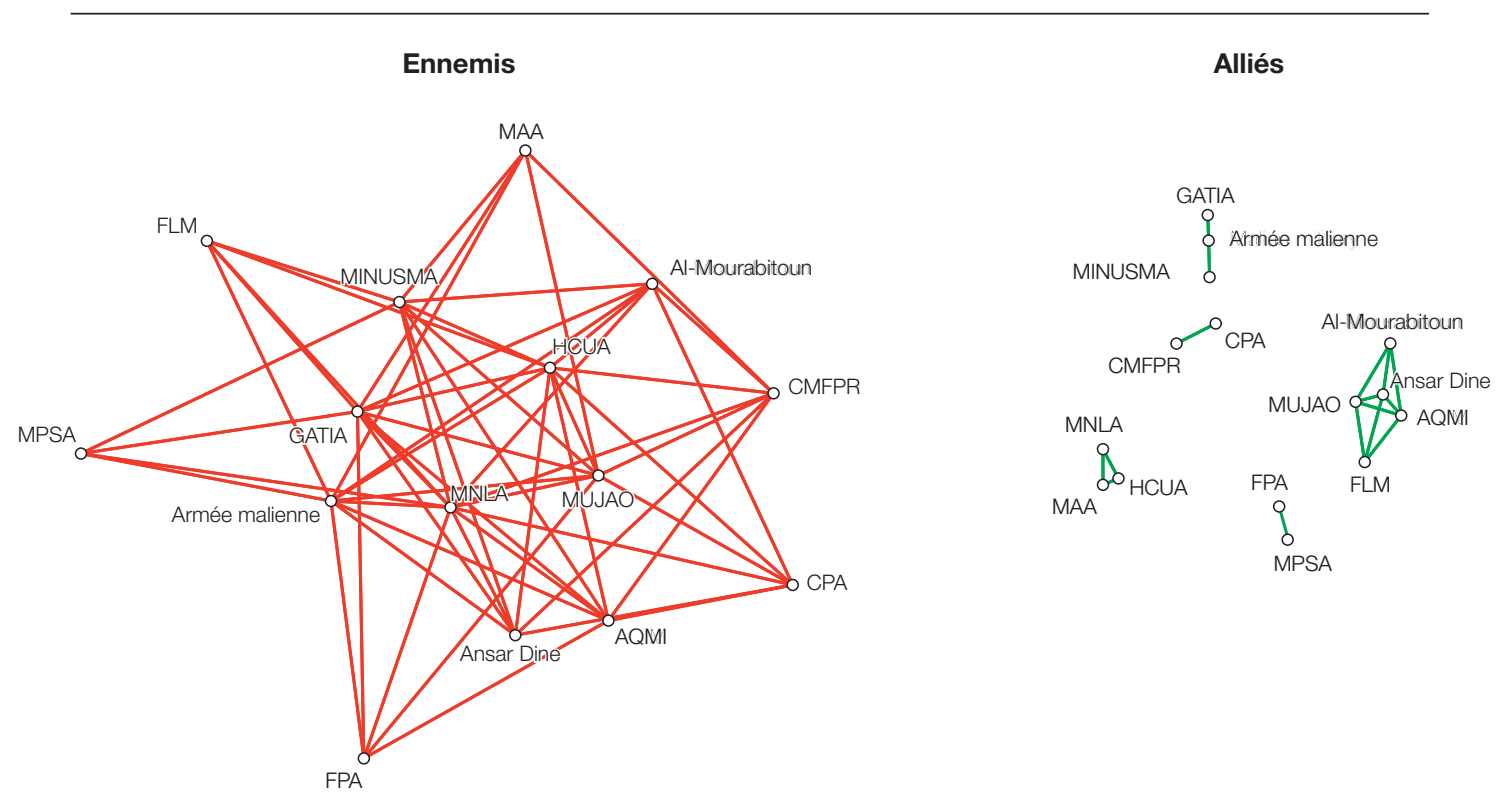

Source : Walther et Tisseron 2015, reproduit avec l'autorisation de The Broker.

L'absence de coalition durable dans la région résulte du fait que les alliances entre groupes se forment et se défont au gré des rapports de force entre parties en conflit. Lors de l'offensive française de 2013, par exemple, de nombreux combattants d'Ansar Dine rejoignent le MNLA ou le Mouvement islamique de l'Azawad (MIA) créé par Alghabass Ag Intalla, un Touareg Ifoghas ayant successivement combattu au sein du MNLA et d'Ansar Dine. Quelques mois plus tard, le MIA intègre le Haut Conseil pour l'unité de l'Azawad (HCUA) dirigé par Mohamed Ag Intalla, le frère d'Alghabass, afin de participer aux négociations de paix. Alors que ces dernières mènent fréquemment à la création de coalitions temporaires, la signature des accords de paix, elle, conduit souvent à une fragmentation des groupes, qui se fracturent à la disparition d'un ennemi commun. Outre ces facteurs politiques, les trafics d'armes, de cigarettes et de drogues sont également une source de rivalités entre groupes, particulièrement le long des axes transsahariens.

Une très grande volatilité caractérise également l'allégeance des soldats et des dirigeants militaires, qui changent fréquemment de camp selon la bonne fortune des forces gouvernementales, des rebelles ou des extrémistes religieux. Provenant d'une famille touareg noble Ifoghas de la région de Kidal, Iyad Ag Ghaly, le chef actuel d'Ansar Dine travaille successivement comme mercenaire dans la Légion islamique du Colonel Kadhafi, rebelle, négociateur pour le gouvernement malien et diplomate en poste en Arabie saoudite avant de chercher à prendre le contrôle du MNLA. Né dans une famille touareg vassale du nord du Mali, le brigadier général El Hadj Ag Gamou connaît un parcours presque aussi sinueux : ayant lui aussi combattu en Libye dans la Légion islamique, il revient ensuite au Mali comme rebelle, avant de rejoindre l'armée malienne et de fonder un mouvement d'autodéfense progouvernemental, le GATIA. 


\section{Des politiques identitaires exclusives}

Nombre de conflits contemporains au Sahara-Sahel n'apparaissent pas principalement motivés par des considérations géopolitiques ou idéologiques, mais par des revendications identitaires. Dans son ouvrage New Wars and Conflicts (2012), Mary Kaldor parle à ce propos de ( politiques identitaires » pour qualifier les luttes armées reposant sur l'appartenance ethnique, raciale ou religieuse. Ces mouvements sont favorisés par la progressive désillusion dans les régimes séculaires postcoloniaux qui suivent la fin de la guerre froide. Contrairement aux mouvements d'inspiration maoïstes dont le fondement était de transformer la paysannerie en une force d'action nouvelle transcendant les divisions plus anciennes, les mouvements basés sur l'identité promeuvent une vision idéalisée du passé, comme celle du Califat portée par l’État islamique ou Boko Haram.

Cette vision politique, qui s'appuie sur la peur, la haine et la destruction, mobilise des idées extrêmes visant à créer des espaces homogènes du point de vue ethnique ou religieux. La destruction de neuf mausolées et de la porte de la mosquée de Sidi Yahia à Tombouctou par des extrémistes d'Ansar Dine en 2012 entre dans cette logique. Conduite quelques jours après que l'UNESCO ait classé la ville sur sa liste du patrimoine de l'humanité en péril, cette initiative, qui laisse stupéfaite aussi bien la population locale que la communauté internationale, vise à désacraliser toutes les manifestations religieuses considérées comme non orthodoxes par les extrémistes. Elle rappelle la destruction des bouddhas de Bamiyan par les Talibans en 2001 et annonce la destruction organisée du patrimoine culturel menée par l’État islamique en Irak, Libye et Syrie à partir de 2014.

Outre les attaques contre le patrimoine culturel, l'une des conséquences les plus dramatiques de ces politiques identitaires est de considérer les civils comme les principales cibles des conflits, non comme des victimes collatérales. Ceci n'est pas spécifique au Sahara-Sahel ; l'essentiel des victimes civiles des conflits africains est en effet volontairement pris pour cible par les belligérants. Cette volonté délibérée de tuer ou de faire fuir les civils pour créer des espaces homogènes du point de vue ethnique ou religieux, distingue les insurrections contemporaines d'Afrique subsaharienne des guérillas plus anciennes, dont l'objectif était de gagner les faveurs de la population locale, sans laquelle les insurgés ne pouvaient pas survivre. Elle illustre le fait que l'objectif des guerres et conflits contemporains du Sahel et du Sahara n'est pas de défendre un territoire, mais de contrôler sa population. Comme le résume le Général Linder, ancien commandant du Special Operations Command-Africa, ( In Africa, it's never about seizing terrain ) (cité dans Griswold, 2014), pour signifier l'impossibilité de contrôler militairement l'ensemble du territoire.

Le conflit malien a, de ce point de vue, fait la démonstration que les rebelles indépendantistes et les extrémistes religieux étaient bien moins intéressés à exercer leur pouvoir sur l'ensemble du nord du Mali - que les rebelles appellent Azawad - que d'occuper un certain nombre de villes et d'axes transsahariens stratégiques nécessaires au contrôle de la population (Retaillé et Walther, 2013). Ces villes sont avant tout Kidal et Tessalit, dans l'extrême nord-est malien, puis Gao, Bourem et Tombouctou dans la boucle du Niger (Carte 7).

Les violences liées aux batailles entre forces gouvernementales, rebelles, milices et communautés ethniques organisées sont la première cause de mortalité des conflits pendant la majeure partie des 20 dernières années en Afrique de l'Ouest et du Nord, suivie de très près par les violences exercées contre les civils (Figure 4). Une évolution similaire est observée dans les pays du G5 (Burkina Faso, Mali, Mauritanie, Niger et Tchad) principalement situés en zone sahélo-saharienne. La violence à distance qui résulte de l'usage de bombes, d'engins explosifs improvisés, de mortiers ou de missiles, et les émeutes et manifestations font comparativement moins de victimes. 
Carte 7

L'insurrection de l'Azawad, 2012

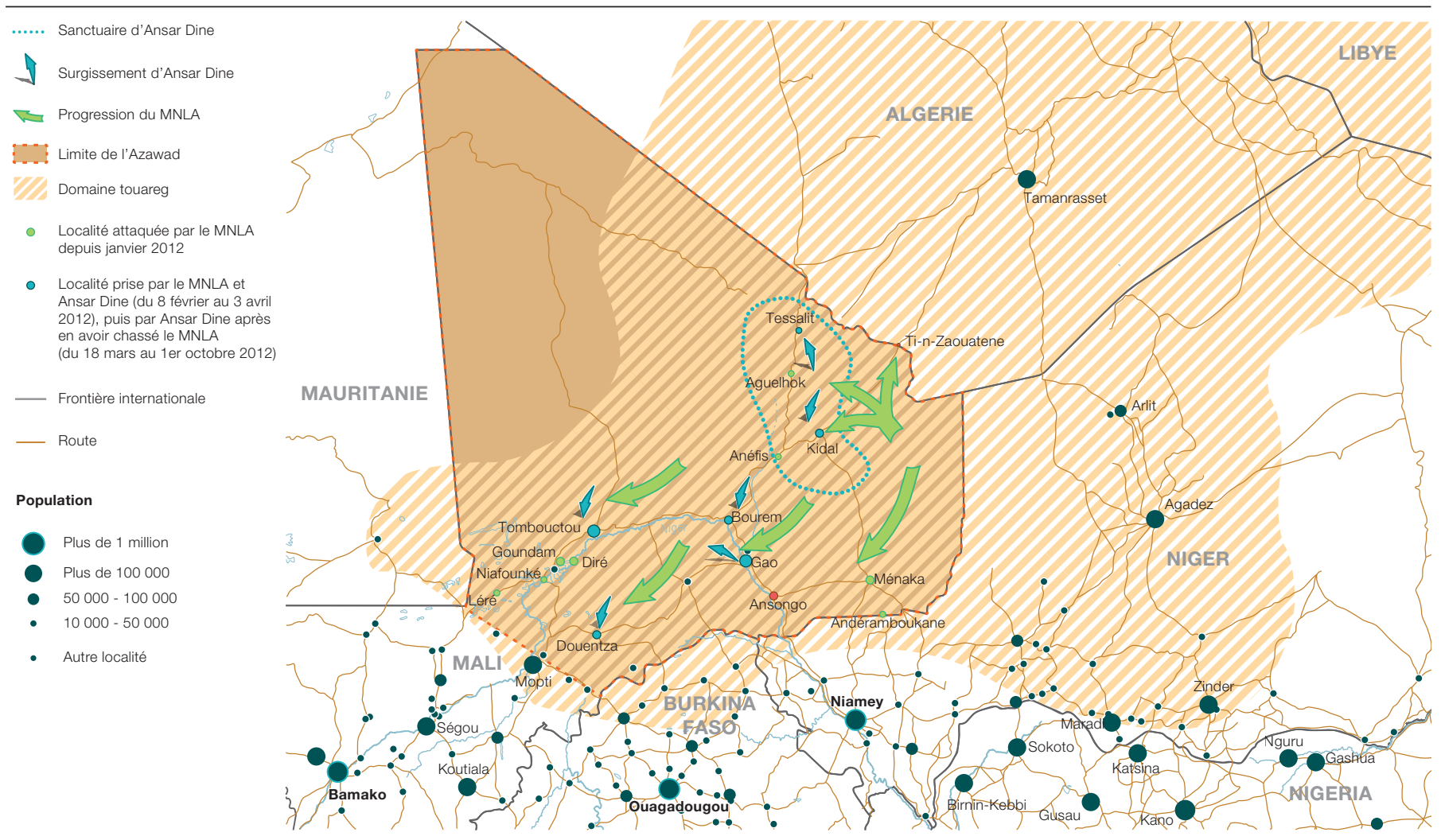

Source : OCDE/CSAO, 2014

Figure 4

Victimes par type de violence, Afrique de l'Ouest et du Nord, 1997-2016

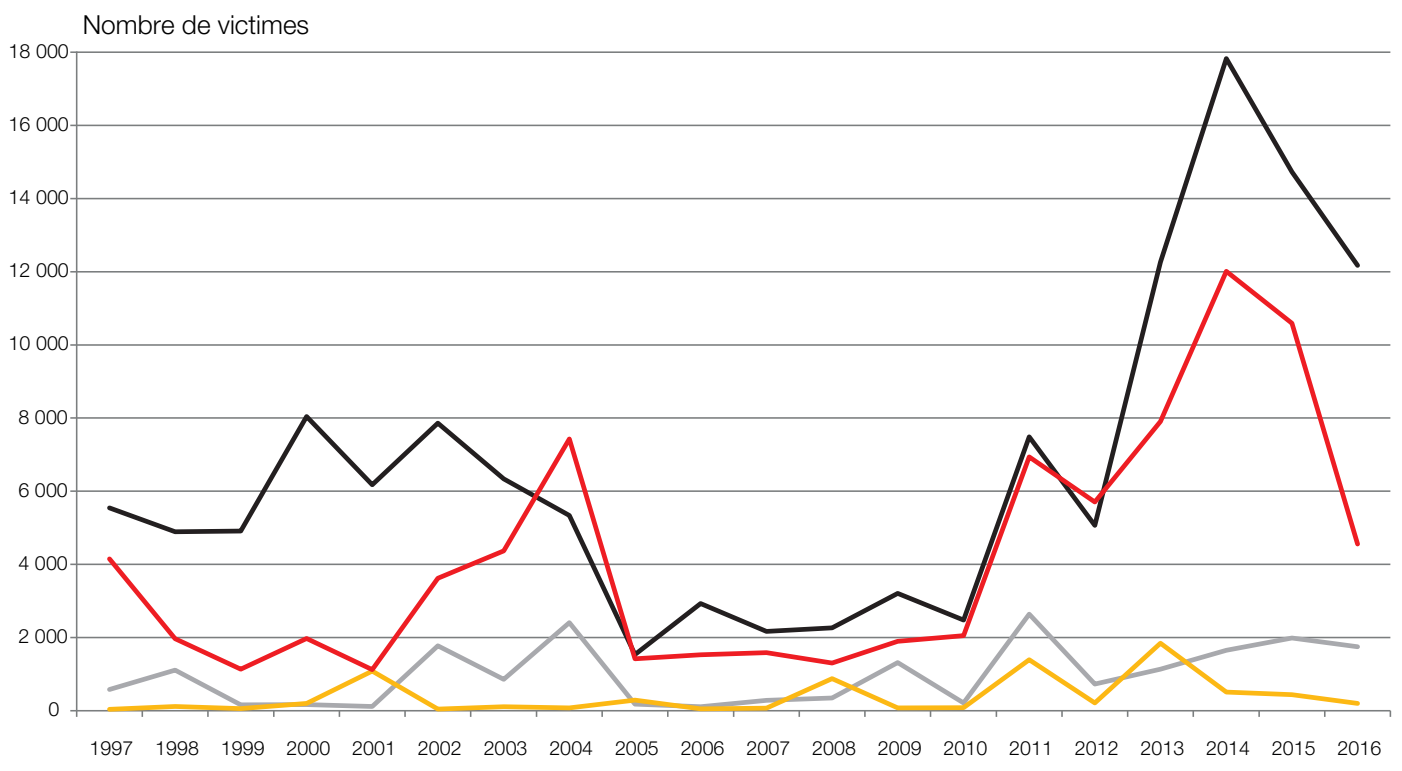

— Batailles - Violences contre civils Uiolence à distance Émeutes/révolutions

Source : ACLED, données 1997-2016 (www.acleddata.com/data/africa) 
Les victimes civiles comptabilisées ici résultent des violences exercées par les gouvernements, les rebelles, les milices et les émeutiers à l'encontre de populations non armées (Raleigh et Dowd, 2015). Elles ne comptabilisent cependant pas les victimes succombant des suites des maladies, de la malnutrition et des déplacements forcés liés aux conflits. Souvent différés, ces effets secondaires augmentent considérablement le prix payé par les civils dans les conflits africains. D’une manière générale en Afrique de l'Ouest, les grands foyers d'insécurité nutritionnelle sont également des régions de conflits (PREGEC, 2017), qu'il s'agisse du Liptako-Gourma, du nord du Mali ou de la région du lac Tchad, où l'insurrection de Boko Haram a forcé plus de 220000 personnes à trouver refuge dans les pays voisins et occasionné plus de 2.3 millions de déplacés internes (HCR, 2017, voir aussi Banque mondiale et HCR, 2016).

\section{Des groupes armés mobiles}

Du point de vue militaire, les protagonistes des conflits du Sahel et du Sahara ont tendance à éviter la confrontation directe avec les forces régulières. Les conflits sahélo-sahariens modernes sont habituellement marqués par une succession d'incidents peu concluants qui mobilisent un nombre restreint de combattants et qui peuvent intervenir à plusieurs centaines de kilomètres de distance. Ces caractéristiques renforcent l'impression que les conflits sahélo-sahariens ne connaissent ni début ni fin et affectent, sans logique apparente, l'ensemble de la région. Investissant aussi peu que possible dans des équipements lourds, les combattants irréguliers compensent leurs faibles effectifs par une grande mobilité. T.E. Lawrence (1920) avait déjà remarqué ce principe en Arabie en affirmant que dans le désert, (c l'espace est plus grand que le pouvoir des armées ») et suggéré que les principes de la guerre dans le désert sont comparables à ceux de la guerre navale. En mer comme dans le désert, les insurgés sont mobiles, relativement indépendants de bases fixes et indifférents aux contraintes de leur environnement.

Le conflit malien a, de ce point de vue, fait la preuve que les moyens militaires des rebelles et des extrémistes religieux ne pouvaient rivaliser avec ceux d'une armée moderne. Pour avoir oublié ce principe fondamental des conflits asymétriques et concentré un grand nombre de leurs pick-up en vue de leur offensive sur Mopti, les extrémistes d'Ansar Dine, du MUJAO et d'AQMI subissent une défaite très rapide dans les premières semaines de janvier 2013 (Chivvis, 2015). Durant l'Opération Serval qui conduisit à la reconquête du nord du Mali, les rebelles du MNLA et les extrémistes religieux, au contraire, n'opposèrent que peu de résistance, préférant fuir plutôt que combattre les forces françaises. Tombouctou, Gao et Kidal, pourtant le fief historique des rébellions touareg, sont reconquises avec un minimum de combats. C'est surtout dans la vallée de l'Ametetai, au sud-est de Tessalit, que le conflit malien prend la forme d'une confrontation directe entre les forces françaises et tchadiennes d'un côté, et les combattants d'AQMI de l'autre. Depuis lors, la plupart des confrontations militaires s'illustrent par des attaques suicides ou par des engins explosifs improvisés visant les convois de la force multinationale ou les bases de l'armée malienne.

\section{QUELLES RÉPONSES INSTITUTIONNELLES ?}

D ans les sections précédentes, cette note analyse les particularités de la situation sécuritaire du Sahel et du Sahara, aujourd'hui confrontés à une instabilité politique exceptionnelle mêlant rébellions, insurrections jihadistes, coups d'État, mouvements contestataires et trafics illicites. Elle montre que la région n'est pas seulement victime d'une intensification des guerres et des conflits qui marquent le $\mathrm{XX}^{\mathrm{e}}$ siècle, mais qu'elle est aussi devenue le théâtre d'opérations de trois types de violence organisée : les luttes armées dont la finalité est politique, les activités criminelles motivées par l'enrichissement personnel et les violations des droits humains. Cette combinaison de violences s'exprime aujourd'hui dans un environnement sécuritaire mondialisé, qui ne connaît plus de frontières strictes entre le local et le global, la sphère domestique et la sphère internationale, le militaire et le civil, la politique et l'identité. 
Les solutions militaires et civiles mises en œuvre par les États de la région et leurs partenaires internationaux doivent tenir compte du fait que les luttes armées s'inscrivent dans un environnement mondialisé qui redéfinit l'échelle des conflits et les relations entre belligérants.

La dégradation de la situation sécuritaire au cours des 15 dernières années montre que les solutions militaires doivent être définies et appliquées sur une base régionale. Le conflit malien a de ce point de vue servi de catalyseur à de nombreuses initiatives destinées à promouvoir la sécurité, la gouvernance et le développement dans la région. Ces efforts reçoivent le soutien du Conseil de sécurité en juin 2017 et culminent avec la mise en place d'une force militaire conjointe par les États du G5. L'Alliance pour le Sahel nouvellement créée devrait contribuer à coordonner les initiatives, jusqu'ici dispersées, des principaux bailleurs dans la région.

En parallèle, il apparaît également que le règlement des conflits à long terme doit passer par une restauration de la légitimité des États, minée (c par le bas » par la multiplication de groupes armés. Les agendas contradictoires et les alliances imprévisibles de ces groupes conduisent ainsi à brouiller la distinction habituelle entre le gouvernement, l'armée nationale et la société qui constituait jusqu'alors l'un des piliers des États modernes de la région. Au cours des dernières décennies, les interventions militaires et les procédures judiciaires internationales se sont aussi souvent substitués (( par le haut)) aux États lorsque ces derniers étaient incapables de défendre leur territoire, de maintenir l'ordre intérieur ou de protéger leur patrimoine culturel. Dans le cas le plus dramatique, au Mali, les forces armées nationales sont dans l'incapacité opérationnelle de défendre le territoire national et de maintenir l'ordre intérieur sans le soutien des quelque 15000 soldats de la mission des Nations Unies et de la France et de répondre aux destructions de leur patrimoine culturel sans le recours de la CIJ. Enfin, la légitimité des États sahélo-sahariens est soumise à une compétition accrue de la part des organisations non gouvernementales dont les actions, bien que nourries par des motivations humanitaires, se substituent « par le côté ») aux services publics, notamment lors de conflits.

Le règlement des conflits dans la région nécessite aussi que des solutions de gouvernance inclusives soient trouvées au sein de chaque État. Le fait que les civils paient un prix si important dans les conflits actuels devraient inciter les pouvoirs publics à recourir à la force minimum nécessaire dans leurs opérations anti-insurrectionnelles. Une stratégie visant à protéger les populations civiles des violences et à gagner leur soutien est la plus à même de contrecarrer les stratégies des groupes extrémistes fondées sur la peur et l'exclusion. Cette stratégie peut passer par la mise en place de régions sécurisées, dans lesquelles des formes alternatives de politique plus inclusives sont mises en place, par l'établissement d'un dialogue entre les États et les acteurs locaux reconnus pour leur intégrité plutôt que leur opportunisme, et par des initiatives de réhabilitation des rebelles et extrémistes religieux. Plus généralement, le règlement des conflits doit passer par la reconnaissance étatique des vertus de la diversité et du cosmopolitanisme, deux ingrédients indispensables pour lutter contre les politiques identitaires.

\section{NOTES}

1 Les guerres se caractérisent par des luttes armées entre États et/ou acteurs non étatiques. Elles débutent par une déclaration de guerre et se terminent par une convention signalant officiellement la fin des hostilités (traité de paix, capitulation). Les conflits caractérisent un état prolongé de lutte entre personnes, organisations, ou pays, sans déclaration formelle de début et de fin.

2 La région considérée ici couvre les 15 pays de la Communauté économique des États de l'Afrique de l'Ouest (CEDEAO) auxquels s'ajoutent la Mauritanie, le Cameroun, le Tchad, le Soudan et le Soudan du Sud. En Afrique du Nord, les données couvrent le Maroc, le Sahara occidental, l'Algérie, la Tunisie, la Libye et l'Égypte. Le Sahara-Sahel n'est pas quant à lui défini comme un ensemble de pays, mais comme un espace de circulation ouvert sur les villes méditerranéennes au nord et sur les marchés et espaces de production ouest-africains au sud.

3 Boko Haram, dont l'étymologie abrégée en haoussa est contestée, s'appelle lui-même Jamā'at Ahl as-Sunnah lid-Da'wah wa'l-Jihād (Groupe sunnite pour la prédication et le jihad) depuis 2010 et al-Wilāyat al-Islāmiyya Gharb Afrīqiyyah (État islamique, province d'Afrique de l'Ouest) depuis son serment d'allégeance en 2015. 


\section{RÉFÉRENCES}

ACLED (2016), Armed Conflict Location and Event Data, données 1997-2016, version 7 (www.acleddata.com/data/ africa).

Banque mondiale et HCR (2016), "Forced Displacement by the Boko Haram conflict in the Lake Chad region ", Genève et Washington, D.C., https://data2.unhcr.org/en/documents/details/52535 (consulté le 12 juillet 2017).

Barkindo, F.A. (2016), " How Boko Haram exploits history and memory ", Africa Research Institute, Londres, https://www.africaresearchinstitute.org/newsite/publications/boko-haram-exploits-history-memory/ (consulté le 12 juillet 2017).

Bencherif, A. et A. Campana (2016), "Alliances of convenience: Assessing the dynamics of the Malian insurgency ", Mediterranean Politics, vol. 22, n¹, pp. 115-134.

Chivvis, C.S. (2015), The French War on Al Qa'ida in Africa, Cambridge University Press, Cambridge.

Daniel, S. (2012), AQMI. L'industrie de l'enlèvement, Fayard, Paris.

Département d'État des États-Unis (2017), Foreign Terrorist Organizations, Washington, DC, https://www.state. gov/j/ct/rls/other/des/123085.htm

Dowd, C. (2018), " Nigeria's Boko Haram: Local, national and transnational dynamics ", in Walther, O. et W. Miles (dir. pub.) African Border Disorders, Routledge, Abingdon, pp. 115-135.

Gartenstein-Ross, D. et J. Zenn (2016), "Boko Haram's doomed marriage to the Islamic State ", War on the Rocks, 26 août, https://warontherocks.com/2016/08/boko-harams-doomed-marriage-to-the-islamic-state/ (consulté le 12 juillet 2017).

Global Terror Watch (2017), Groupes armés, liste par pays, Genève, www.globalterrorwatch.ch/index.php/ groupes-armes-liste-par-pays.

Grégoire, E. (2013), «Islamistes et rebelles touaregs maliens : Alliances, rivalités et ruptures », EchoGéo, http://echogeo.revues.org/13466 (consulté le 12 juillet 2017).

Griffin, C. (2016), "Operation Barkhane and Boko Haram: French counterterrorism and military cooperation in the Sahel ", Small Wars \& Insurgencies, vol. 27, n5, pp. 896-913.

Griswold, E. (2014), "Can General Linder's Special Operations Forces stop the next terrorist threat? " The New York Times, 13 juin.

Harmon, S.A. (2014), Terror and Insurgency in the Sahara-Sahel Region, Ashgate, Farnham.

HCR (2017), « Nigeria situation », https://data2.unhcr.org/en/situations/nigeriasituation (consulté le 12 juillet 2017).

ICG (2016), « Boko Haram on the back foot? »International Crisis Group Africa Briefing 120.

Kaldor, M. (2012), New and Old Wars. Organized Violence in a Global Area, Stanford University Press, Palo Alto.

Knoke, D. (2013), « It takes a network: The rise and fall of social network analysis in U.S. Army counterinsurgency doctrine ", Connections, vol. 33, n¹, pp. 1-8.

Lawrence, T.E. (1920), « The evolution of a revolt ", Army Quarterly and Defense Journal, Octobre, http://usacac. army.mil/cac2/cgsc/carl/download/csipubs/lawrence.pdf(consulté le 12 juillet 2017).

Marsh, N. (2017), "Brothers came back with weapons. The effects of arms proliferation from Libya ", PRISM, vol. $6, n^{\circ} 4$, pp. $79-96$.

Melander, E., T. Pettersson et L. Themnér (2016), « Organized violence, 1989-2015 », Journal of Peace Research, vol. 53, n5, pp. 727-742.

OCDE/CSAO (2014), Un atlas du Sahara-Sahel : Géographie, économie et insécurité, Éditions OCDE, Paris, http://dx.doi.org/10.1787/9789264222335-fr.

PREGEC (2017), " Avis sur la situation alimentaire et nutritionnelle (juin-août) et perspectives de la campagne agropastorale 2017-18, Praia, Dispositif régional de prévention et de gestion des crises alimentaires ", www.oecd.org/fr/sites/rpca/documents/RPCA_Avis_PREGEC_Juin2017_FR.pdf (consulté le 12 juillet 2017).

Raleigh, C., A. Linke, H. Hegre et J. Karlsen (2010), «Introducing ACLED-Armed Conflict Location and Event Data », Journal of Peace Research, vol. 47, n5, pp. 651-660.

Raleigh, C. et C. Dowd (2015), "Armed Conflict Location and Event Data Project (ACLED) Codebook », https:// www.acleddata.com/wp-content/uploads/2015/01/ACLED_Codebook_2015.pdf (consulté le 12 juillet 2017).

Retaillé, D. et O. Walther (2013), "Conceptualizing the mobility of space through the Malian conflict ", Annales de Géographie, vol. 6, pp. 595-618. 
Nations Unies (2017), Security Council Welcomes Deployment of Joint Force to Combat Terrorism Threat, "Transnational Crime in Sahel, Unanimously Adopting Resolution 2359 », Nations Unies, New York, https://www.un.org/press/en/2017/sc12881.doc.htm (consulté le 12 juillet 2017).

Walther, O. et A. Tisseron (2015), "Strange bedfellows: a network analysis of Mali's northern conflict ", The Broker, 18 décembre, www.thebrokeronline.eu/Articles/Strange-bedfellows-a-network-analysis-of-Mali-snorthern-conflict (consulté le 12 juillet 2017).

Walther, O. et W. Miles (dir. pub.) (2018), African Border Disorders. Addressing Transnational Extremist Organizations, Routledge, Abingdon.

Walther, O., C. Leuprecht et D. Skillicorn (2018), « Networks and spatial patterns of extremist organizations in North and West Africa ", in Walther, O. et W. Miles (dir. pub.), African Border Disorders. Routledge, Abingdon, pp. 60-86

Williams, P. (2011), War and Conflict in Africa, Polity Press, Cambridge. 
ANNEXE : GROUPES ARMÉS EN CONFLIT OUVERT AVEC LES ÉTATS SAHÉLO-SAHARIENS, JANVIER 1990-DÉCEMBRE 2016

\begin{tabular}{|c|c|}
\hline Pays & Groupes armés \\
\hline Algérie & $\begin{array}{l}\text { Al-Qaida } \\
\text { Al-Qaida au Maghreb islamique (AQMI) } \\
\text { Al Moulathamoun (Signataires par le sang) } \\
\text { Al-Mourabitoun (les Almoravides) } \\
\text { Ansar Dine } \\
\text { Armée et Front islamique du salut (AIS, FIS) } \\
\text { Brigade Al Farouk } \\
\text { Brigade Ennour } \\
\text { Groupe islamique armé (GIA) } \\
\text { Groupe salafiste pour la prédication et le combat (GSPC) } \\
\text { Groupe salafiste libre (GSL) } \\
\text { Humat al-Da'wa al-Salafiya } \\
\text { Jama'at al-Muslimiin } \\
\text { Ligue islamique pour la dawa et le jihad (LIDD) } \\
\text { Mouvement pour l'unicité et le jihad en Afrique de l'Ouest (MUJA0) } \\
\text { Mouvement islamique de l'Azawad (MIA) } \\
\text { Brigade Okba Ibn Nafaa } \\
\text { Salafia Jihadia } \\
\text { Soldats du califat } \\
\text { Brigade Timizart }\end{array}$ \\
\hline Burkina Faso & $\begin{array}{l}\text { Ansaroul Islam } \\
\text { Al-Qaida au Maghreb islamique (AQMI) } \\
\text { Al-Mourabitoun (les Almoravides) } \\
\text { État islamique (Sahara) }\end{array}$ \\
\hline Libye & $\begin{array}{l}\text { Brigade des martyrs d'Abu Salim } \\
\text { Ajdabiya Revolutionaries Shura Council } \\
\text { Al Moulathamoun (Signataires par le sang) } \\
\text { Al-Mourabitoun (les Almoravides) } \\
\text { Al-Qaida } \\
\text { Al-Qaida au Maghreb islamique (AQMI) } \\
\text { Brigade AI Qaqa } \\
\text { Brigade Al-Kanni } \\
\text { Ansar al-Sharia } \\
\text { Barqa Youth Movement } \\
\text { Brigade des martyrs de Brega } \\
\text { Brigades de defense de Benghazi } \\
\text { Conseil national de transition (NTC) } \\
\text { Brigade Al-Farouk } \\
\text { Brigade des martyrs du 17 février } \\
\text { Fighters of The Martyrs Brigade } \\
\text { Free Libya Martyrs Brigade } \\
\text { Groupe islamique combattant en Libye (LIFG) } \\
\text { Harakat al-Shuhada'a al-Islamiyah } \\
\text { Jaysh al-Mujahidin } \\
\text { État islamique (Libye) } \\
\text { Émirat islamique de Barqa } \\
\text { Libya Dawn } \\
\text { Libya Shield } 1 \\
\text { Libyan Rebel Forces } \\
\text { Brigade Rafallah al-Sahati } \\
\text { Shura Council of Benghazi Revolutionaries } \\
\text { Shura Council of Islamic Youth }\end{array}$ \\
\hline
\end{tabular}




\begin{tabular}{|c|c|}
\hline Pays & Groupes armés \\
\hline Mali & $\begin{array}{l}\text { Alliance démocratique du } 23 \text { mai pour le changement (ADC) } \\
\text { Alliance touareg Nord Mali pour le changement (ATNMC) } \\
\text { Al Moulathamoun (Signataires par le sang) } \\
\text { Al-Mourabitoun (les Almoravides) } \\
\text { Al-Qaida au Maghreb islamique (AQMI) } \\
\text { Ansar Dine } \\
\text { Ansar al Sharia (Mali) } \\
\text { Coalition du peuple pour l'Azawad (CPA) } \\
\text { Congrès pour la justice dans l'Azawad (CJA) } \\
\text { Coordination des mouvements de l'Azawad (CMA) } \\
\text { Front de libération du Macina } \\
\text { Front islamique arabe de l'Azawad (FIAA) } \\
\text { Groupe salafiste pour la prédication et le combat (GSPC) } \\
\text { Haut Conseil pour l'unité de l'Azawad (HCUA) } \\
\text { Jama'a Nusrat ul-Islam wa al-Muslimin' (JNIM) } \\
\text { Mouvement arabe de l'Azawad (MAA) } \\
\text { Mouvement populaire de l'Azawad (MPA) } \\
\text { Mouvement pour le salut de l'Azawad (MSA) } \\
\text { Mouvement national de libération de l'Azawad (MNLA) } \\
\text { Mouvement pour l'unicité et le jihad en Afrique de l'Ouest (MUJAO) }\end{array}$ \\
\hline Mauritanie & $\begin{array}{l}\text { Al-Qaida au Maghreb islamique (AQMI) } \\
\text { Groupe salafiste pour la prédication et le combat (GSPC) }\end{array}$ \\
\hline Maroc & $\begin{array}{l}\text { Al-Qaida au Maghreb islamique (AQMI) } \\
\text { Groupe islamique combattant marocain (GICM) } \\
\text { Salafia Jihadia }\end{array}$ \\
\hline Niger & $\begin{array}{l}\text { Al-Qaida au Maghreb islamique (AQMI) } \\
\text { Boko Haram } \\
\text { Front de libération de l'Aïr et de l'Azawad (FLAA) } \\
\text { Coordination de la résistance armée (CRA) } \\
\text { Front démocratique du renouveau (FDR) } \\
\text { Forces armées révolutionnaires du Sahara (FARS) } \\
\text { Groupe salafiste pour la prédication et le combat (GSPC) } \\
\text { Mouvement des Nigériens pour la justice (MNJ) }\end{array}$ \\
\hline $\begin{array}{l}\text { Nigéria } \\
\text { (nord-est) }\end{array}$ & $\begin{array}{l}\text { Ansaru } \\
\text { Boko Haram }\end{array}$ \\
\hline
\end{tabular}





\section{DANS LA MÊME COLLECTION :}

Allen, T. et P. Heinrigs (2016), « Les nouvelles opportunités de l'économie alimentaire ouest-africaine», Notes ouest-africaines, $\mathrm{N}^{\circ} 1$, Éditions OCDE, Paris. http://dx.doi.org/10.1787/5jlwjg67l25f-fr

Lewis, K. et C. Buontempo (2016), « Climate Impacts in the Sahel and West Africa: The Role of Climate Science in Policy Making ), Notes ouest-africaines, N², Éditions OCDE, Paris. http://dx.doi.org/10.1787/5jlsmktwjcd0-en

Gnisci, D. (2016), “ Women's Roles in the West African Food System: Implications and Prospects for Food Security and Resilience »), Notes ouest-africaines, №3, Éditions OCDE, Paris. http://dx.doi.org/10.1787/5jlpl4mh1hxn-en

Staatz, J. et F. Hollinger (2016), « West African Food Systems and Changing Consumer Demands », Notes ouest-africaines, $N^{\circ} 4$, Éditions OCDE, Paris. http://dx.doi.org/10.1787/b165522b-en

Prieto Curiel, R., P. Heinrigs et I. Heo (2017), “ Cities and Spatial Interactions in West Africa: A Clustering Analysis of the Local Interactions of Urban Agglomerations », Notes ouest-africaines, $N^{\circ} 5$, Éditions OCDE, Paris. http://dx.doi.org/10.1787/57b30601-en

Walther, O. (2017), ( Les réseaux de la coopération transfrontalière en Afrique de l'Ouest », Notes ouest-africaines, Nº6, Éditions OCDE, Paris. http://dx.doi.org/10.1787/b7ad4957-fr

Ibrahim, I.Y. (2017), ( Insurrections jihadistes en Afrique de l'Ouest : idéologie mondiale, contexte local, motivations individuelles », Notes ouest-africaines, $\mathrm{N}^{\circ} 07$, Éditions OCDE, Paris. http://dx.doi.org/10.1787/1da64565-fr

Allen, T. (2017), ( Le coût des prix alimentaires élevés en Afrique de l'Ouest », Notes ouestafricaines, $\mathrm{N}^{\circ} 08$, Éditions OCDE, Paris. http://dx.doi.org/10.1787/48e99091-fr

Van Den Hoek, J. (2017), « Agricultural market activity and Boko Haram attacks in northeastern Nigeria ), West African Papers, $\mathrm{N}^{\circ} 09$, OECD Publishing, Paris. http://dx.doi.org/10.1787/13ba9f2e-en

En savoir plus : lia.beyeler@oecd.org 